\title{
MIMO Radar Waveform Optimization With Prior Information of the Extended Target and Clutter
}

\author{
Chun-Yang Chen, Student Member, IEEE, and P. P. Vaidyanathan, Fellow, IEEE
}

\begin{abstract}
The concept of multiple-input multiple-output (MIMO) radar allows each transmitting antenna element to transmit an arbitrary waveform. This provides extra degrees of freedom compared to the traditional transmit beamforming approach. It has been shown in the recent literature that MIMO radar systems have many advantages. In this paper, we consider the joint optimization of waveforms and receiving filters in the MIMO radar for the case of extended target in clutter. A novel iterative algorithm is proposed to optimize the waveforms and receiving filters such that the detection performance can be maximized. The corresponding iterative algorithms are also developed for the case where only the statistics or the uncertainty set of the target impulse response is available. These algorithms guarantee that the SINR performance improves in each iteration step. Numerical results show that the proposed methods have better SINR performance than existing design methods.
\end{abstract}

Index Terms-Beamforming, clutter, extended target, multipleinput multiple-output (MIMO) radar, waveform design.

\section{INTRODUCTION}

$\mathbf{M}$ ULTIPLE-INPUT multiple-output (MIMO) radar is an emerging field which has drawn considerable attention recently. Unlike the traditional SIMO (single-input multipleoutput) radar, which can only transmit scaled versions of a signal waveform, the MIMO radar is capable of transmitting arbitrary waveforms [21], [22]. This provides extra degrees of freedom in the design of the radar systems. In this paper, we consider the joint optimization of the transmitted waveforms and receiving filters in the MIMO radar for the case of extended target in clutter.

The MIMO radar systems can be classified into two categories: 1) bistatic MIMO radar [27], and 2) colocated MIMO radar [28]. In the bistatic case, the transmitting antennas are widely apart such that each views a different aspect of the target. The concept of MIMO radar can be used to increase the spatial diversity of the system [23], [24], [26]. The spatial diversity can improve the performance of detection [26] and angle estimation [25]. A tutorial on the bistatic MIMO radar can be found in [27]. In the colocated radar case, the transmitting antennas are close enough such that the target radar cross sections (RCS) observed by the transmitting antenna elements are identical. In this case,

Manuscript received November 10, 2008; accepted April 03, 2009. First published April 24, 2009; current version published August 12, 2009. The associate editor coordinating the review of this manuscript and approving it for publication was Prof. Andreas Jakobsson. This work was supported in part by the ONR Grant N00014-08-1-0709 and by the California Institute of Technology.

The authors are with the Electrical Engineering Department, California Institute of Technology, Pasadena, CA 91125 USA (e-mail: cyc@ caltech.edu).

Digital Object Identifier 10.1109/TSP.2009.2021632 the concept of MIMO radar can be used to increase the spatial resolution. Several advantages have been demonstrated by different authors, including excellent interference rejection capability [30], [31], improved parameter identifiability [29], and enhanced flexibility for transmit beampattern design [5], [7]. A tutorial on the colocated MIMO radar can be found in [28]. In this paper, we focus on the colocated MIMO radar.

The MIMO radar waveform design problems have been studied in [5]-[16]. These methods can be broken into three categories: 1) covariance matrix based design [5]-[9]; 2) radar ambiguity function based design [10]-[13]; and 3) extended target based design [14]-[16]. In the covariance matrix based design methods, the covariance matrix of the waveforms are considered instead of the entire waveform. Consequently, this kind of design methods affects only the spatial domain. In [5] and [6], the covariance matrix of the transmitted waveforms is designed such that the power can be transmitted to a desired range of angles. In [7], the authors have also designed the covariance matrix of the transmitted waveforms to control the spatial power. However, in [7], the cross correlation between the transmitted signals at a number of given target locations is minimized. This can further increase the spatial resolution in the receiver. In [8], the covariances between waveforms have been optimized for several design criteria based on the Cramér-Rao bound matrix. In [9], given the optimized covariance matrix, the corresponding signal waveforms are designed to further achieve low peak-to-average-power ratio (PAR) and higher range resolution.

The radar ambiguity function based methods optimize the entire waveforms instead of just their covariances. Thus these design methods involve not only the spatial domain but also the range domain. The angle-Doppler-range resolution of the radar system can be characterized by the MIMO radar ambiguity function [38]-[40]. In [11]-[13], the sidelobe of the autocorrelation and the cross correlation between waveforms are minimized. This sharpens the radar ambiguity function. In [10], the waveforms are directly optimized so that a sharper radar ambiguity function can be obtained. Thus the spatial and range resolution of point targets can be improved.

In the extended target based methods also, the entire waveform is considered as in the radar ambiguity function based approaches. However, unlike the ambiguity function based methods which consider the resolutions of point targets, these methods consider the detection or estimation of extended targets. These methods require some prior information about the target and/or clutter impulse response. The extended target based methods have been also studied in the SIMO case [1]-[4], [17]-[20]. In [17], the waveform is optimized to maximize the 
SINR subject to the constraint that the waveform is similar to a desired waveform. This constraint improves the PAR and the range resolution of the waveform. In [18], the optimal radar code which considers detection probability, Doppler frequency estimation accuracy, PAR and the range resolution is proposed. In [19] and [20], the mutual information between the received waveforms and the target impulse response has been optimized by properly designing the transmitting waveforms. This idea has been extended to the MIMO radar case in [15]. The corresponding robust design has also been proposed in [16]. However, in [15]-[20] the effect of the clutter is ignored. In [1]-[4], the clutter impulse response has been considered. In these methods, the SINR has been maximized to improve the detection performance by properly designing the transmitting waveform. Both [1] and [2] have proposed different iterative algorithms to maximize the SINR. In [1], Pillai et al. have proven that in the continuous-time SIMO radar case, the optimal transmitted waveform must be minimum-phase. For the MIMO radar, SINR maximization with both target and clutter information has been considered in [14]. A MIMO extension of the method in [1] and a gradient based method have been proposed in [14] to solve for the transmitted waveforms. Several suboptimal solutions have also been studied in [14].

In this paper, we consider the waveform design problem which maximizes the SINR in the presence of clutter in the colocated MIMO radar case. As shown in [1], [2], and [14], the difficulty of this problem is that the objective function, namely the SINR, is not a convex function of the transmitted waveforms. Moreover, it cannot be easily solved by Lagrange multiplier methods. In [1], [2], and [14], different iterative methods have been developed. In [2], the algorithm guarantees the SINR improves in each iterative step. However, it has not been extended to the MIMO case because the algorithm is based on the symmetry of the SIMO radar ambiguity function [41], which is no longer valid in the MIMO radar case. On the other hand, in [1] and [14], the algorithms work for the MIMO radar case. However they do not guarantee nondecreasing SINR in each iteration step. Consequently, these algorithms cannot guarantee convergence. In this paper, we propose a new algorithm which works in the MIMO radar case and guarantees nondecreasing SINR in each iteration step. The numerical results show that it converges faster and has better SINR performances than the method in [1] and [14]. We also consider the case where only a partial information of the target impulse response is known. This includes the case where only the statistics of the target impulse response are given and the case where only an uncertainty set of the target impulse response is given. The corresponding iterative algorithms have been developed for both cases.

The extended target based waveform design problem is very different from other types of radar waveform design. It requires the knowledge of the clutter statistics and the target impulse response or its statistics. It also requires the transmitted waveforms to adapt to the changing statistics in real-time. Therefore, it is more complicated than other methods. The clutter information can be estimated by previous received signals before the target appears. We assume the impulse response of the target of interest is known. The goal is to design the waveforms which are best suitable for detecting this particular target of interest. However, this assumption may not be practical because the target impulse response depends on the orientation of the target. Therefore, in Section IV, we discuss the case where only the statistics or the uncertainty set of the target impulse response is available. The optimal waveforms for the detection are the waveforms which lead to the greatest SINR. Thus, we consider the problem of maximizing the SINR with the total energy constraint. Some of the important design issues such as constant modulus and range resolution are not considered in this paper. From a practical stand point, it is important that the radar transmitter has a low PAR. Also, the optimal waveforms obtained by the proposed method may not have good performance for range estimation. The waveform design problem which takes into account these important issues will be explored in the future.

The rest of this paper is organized as follows Section II introduces the signal model, formulates the problem and reviews the existing algorithms. Section III proposes the new iterative algorithm for jointly designing the transmitted waveforms and the receiving filters. Section IV proposes the iterative algorithms for random target and uncertain target cases. Section V shows the results of numerical simulations. Finally Section VI concludes the paper.

Notations: Matrices and vectors are denoted by capital letters in boldface (e.g., A). Superscript ${ }^{T}$ and ${ }^{\dagger}$ denote transpose and transpose conjugation, respectively. The expression $(\mathbf{A})_{k, l}$ represents the element of matrix $\mathbf{A}$ located at the $k$ th row and the $l$ th column. The notation $\operatorname{tr}(\mathbf{A})$ denotes the trace of matrix $\mathbf{A}$. The notation $\|\mathbf{A}\|_{F}$ denotes the Frobenius norm of the matrix A. The notation $\measuredangle a$ denotes the angle of the complex number $a$. The notation $\lfloor a\rfloor$ is defined as the largest integer smaller than $a$. The notation $(n \bmod m)$ represents the remainder of division of $n$ by $m$. The notation $\operatorname{vec}(\mathbf{A})$ denotes a vector formed by reshaping the matrix $\mathbf{A}$. For example, for a matrix $\mathbf{A} \in \mathcal{C}^{N \times M}$, the $k$ th element of the vector $\mathbf{x}=\operatorname{vec}(\mathbf{A}) \in \mathcal{C}^{N M \times 1}$ can be expressed as

$$
(\mathbf{x})_{k}=(\mathbf{A})_{(k \bmod N),\lfloor k\rfloor} \text {. }
$$

\section{PRoblem Formulation AND REVIEW}

Fig. 1(a) illustrates the model used in this paper. Consider a MIMO radar system with $N_{T}$ transmitting antennas and $N_{R}$ receiving antennas. A finite duration $N_{T} \times 1$ vector signal $\mathbf{f}(n)$ is converted to analog waveforms, modulated, and emitted. The waveforms are reflected back by the target and clutter with transfer function $\mathbf{T}_{a}(s)$ and $\mathbf{C}_{a}(s)$, respectively. In the receiver, $N_{R}$ waveforms are received, demodulated and converted back to a discrete vector signal $\mathbf{r}(n)$. Then the received signal $\mathbf{r}(n)$ is processed by a receiving filter $\mathbf{H}(z)$ to further determine the existence of the target. Fig. 1(b) illustrates the discrete baseband equivalent model where $\mathbf{T}(z)$ and $\mathbf{C}(z)$ represent the transfer functions of the target and clutter, respectively. We assume $\mathbf{T}(z)$ is a known FIR filter. It can be represented as

$$
\mathbf{T}(z)=\sum_{n=0}^{L} \mathbf{t}(n) z^{-n}
$$




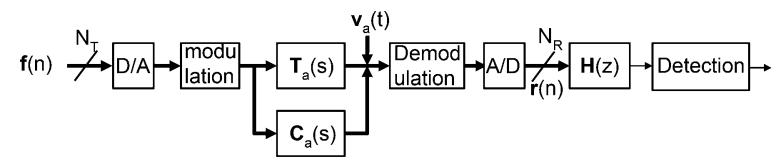

(a)

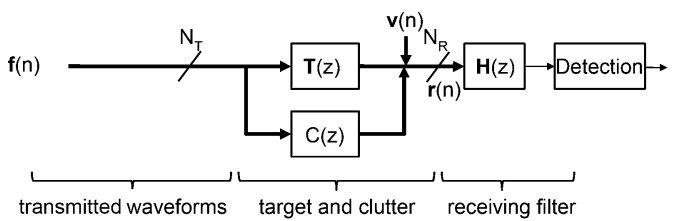

(b)

Fig. 1. Illustration of (a) the signal model. (b) The discrete baseband equivalent model.

where $\mathbf{t}(n) \in \mathbb{C}^{N_{R} \times N_{T}}$ is the impulse response of the target (i.e., $\mathbf{t}_{k, l}(n)=$ impulse response from the $l$ th transmitting antenna to the $k$ th receiving antenna) and $L$ is the order of the FIR filter. The clutter transfer function can be represented as

$$
\mathbf{C}(z)=\sum_{n=-\infty}^{\infty} \mathbf{c}(n) z^{-n}
$$

where $\mathbf{c}(n) \in \mathbb{C}^{N_{R} \times N_{T}}$ is the impulse response of clutter. We assume $\operatorname{vec}(\mathbf{c}(n))$ is a vector wide-sense stationary (WSS) process with known covariance

$$
\mathbf{R}_{c}(m) \triangleq E\left[\operatorname{vec}(\mathbf{c}(n)) \operatorname{vec}(\mathbf{c}(n-m))^{\dagger}\right] .
$$

The $N_{R} \times 1$ vector process $\mathbf{v}(n)$ shown in Fig. 1(b) represents the noise in the receiver. We also assume the covariance

$$
\mathbf{R}_{v}(m) \triangleq E\left[\mathbf{v}(n) \mathbf{v}(n-m)^{\dagger}\right]
$$

is known. The assumption of the availability of this prior information has also been made in [1]-[4], [14].

With the prior information of the target impulse response and the second order statistics of the clutter impulse response and noise, our goal is to jointly design the $N_{T} \times 1$ transmitted vector waveform $\mathbf{f}(n)$ and the $N_{R} \times 1$ receiving filter $\mathbf{H}(z)$ to maximize the detection rate. It is well known that the optimal detection can be obtained by the log-likelihood ratio test [42]. In this case, the detection rate is a nondecreasing function of the SINR. Therefore, our goal becomes to maximize the SINR by choosing $\mathbf{f}(n)$ and $\mathbf{H}(z)$. The single-input single-output (SISO) case of this problem, where $N_{R}=N_{T}=1$, has been studied by DeLong and Hofstetter in 1967 [2]-[4] and more recently by Pillai et al. [1]. Two different types of iterative methods have been proposed for solving this problem. DeLong and Hofstetter's iterative method takes advantage of the symmetry property of the cross ambiguity function. This method guarantees the SINR improves in each iteration step. Nevertheless, the symmetry property cannot be applied in the general MIMO case. Consequently this method cannot be generalized to the MIMO case. On the other hand, Pillai's method has been generalized to the MIMO case by Friedlander [14]. However, this method does not guarantee that the SINR improves in each iteration step. We will soon briefly review this method. In this paper, we propose a new iterative method for optimizing the MIMO radar transceiver. It works in the MIMO case and also guarantees the SINR improves in every iteration step.

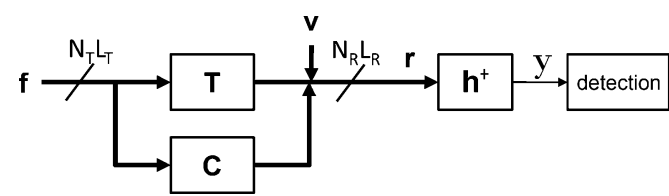

Fig. 2. The FIR equivalent model.

\section{A. Problem Formulation}

The received baseband waveform $\mathbf{r}(n)$ can be expressed as

$$
\mathbf{r}(n)=\sum_{m=0}^{L_{T}}(\mathbf{t}(n-m)+\mathbf{c}(n-m)) \cdot \mathbf{f}(m)+\mathbf{v}(n)
$$

where $L_{T}$ is the order of the finite duration signal $\mathbf{f}(n)$. We define

$$
\mathbf{r} \triangleq\left[\mathbf{r}(0)^{T} \mathbf{r}(1)^{T} \cdots \mathbf{r}\left(L_{R}\right)^{T}\right]^{T} \in \mathbb{C}^{N_{R}\left(L_{R}+1\right) \times 1}
$$

where $L_{R}$ is the order of the receiving filter $\mathbf{H}(z)$. Then the overall received signal can be expressed as

$$
\mathbf{r}=(\mathbf{T}+\mathbf{C}) \mathbf{f}+\mathbf{v}
$$

where

$$
\begin{aligned}
\mathbf{f} \triangleq\left[\mathbf{f}(0)^{T} \mathbf{f}(1)^{T} \cdots \mathbf{f}\left(L_{T}\right)^{T}\right]^{T} & \in \mathbb{C}^{N_{T}\left(L_{T}+1\right) \times 1} \\
\mathbf{v} \triangleq\left[\mathbf{v}(0)^{T} \mathbf{v}(1)^{T} \cdots \mathbf{v}\left(L_{R}\right)^{T}\right]^{T} & \in \mathbb{C}^{N_{R}\left(L_{R}+1\right) \times 1}
\end{aligned}
$$

and $\mathbf{T}$ and $\mathbf{C}$ are block Toeplitz matrices defined as

$$
\mathbf{T} \triangleq\left[\begin{array}{cccc}
\mathbf{t}(0) & \mathbf{0} & \cdots & \mathbf{0} \\
\mathbf{t}(1) & \mathbf{t}(0) & \ddots & \vdots \\
\vdots & \mathbf{t}(1) & \ddots & \mathbf{0} \\
\mathbf{t}(L) & \vdots & \ddots & \mathbf{t}(0) \\
\mathbf{0} & \mathbf{t}(L) & \ddots & \mathbf{t}(1) \\
\vdots & \ddots & \ddots & \vdots \\
\mathbf{0} & \cdots & \mathbf{0} & \mathbf{t}(L)
\end{array}\right]
$$

and

$$
\mathbf{C} \triangleq\left[\begin{array}{cccc}
\mathbf{c}(0) & \mathbf{c}(-1) & \cdots & \mathbf{c}\left(-L_{T}\right) \\
\mathbf{c}(1) & \mathbf{c}(0) & \ddots & \vdots \\
\vdots & \mathbf{c}(1) & \ddots & \vdots \\
\vdots & \ddots & \ddots & \mathbf{c}(0) \\
\vdots & \ddots & \ddots & \mathbf{c}(1) \\
\vdots & \ddots & \ddots & \vdots \\
\mathbf{c}\left(L_{R}\right) & \mathbf{c}\left(L_{R}-1\right) & \cdots & \mathbf{c}(L)
\end{array}\right] .
$$

Fig. 2 illustrates the FIR equivalent model, where the $N_{R} \times$ $\left(L_{R}+1\right)$ vector $\mathbf{h}$ consists of the impulse response of the receiving filter $\mathbf{H}(z)$. The receiving filter output can be expressed as

$$
\mathbf{y}=\mathbf{h}^{\dagger} \mathbf{r}=\underbrace{\mathbf{h}^{\dagger} \mathbf{T f}}_{\text {signal }}+\underbrace{\mathbf{h}^{\dagger} \mathbf{C f}}_{\text {clutter }}+\underbrace{\mathbf{h}^{\dagger} \mathbf{v}}_{\text {noise }} .
$$

Thus the SINR at the filter output can be expressed as

$$
\rho(\mathbf{f}, \mathbf{h}) \triangleq \frac{\left|\mathbf{h}^{\dagger} \mathbf{T f}\right|^{2}}{E\left[\left.\mathbf{h}^{\dagger} \mathbf{C} \mathbf{f}\right|^{2}\right]+E\left[\left|\mathbf{h}^{\dagger} \mathbf{v}\right|^{2}\right]} .
$$


Our goal is to maximize the SINR subject to the power constraint, that is

$$
\max _{\mathbf{f}, \mathbf{h}} \rho(\mathbf{f}, \mathbf{h}) \text { subject to }\|\mathbf{f}\|^{2} \leq 1 .
$$

One can first observe that this problem is in general not convex because the objective function is a fourth order rational function. In general, there will be multiple local maxima in the feasible set. It is in general not easy to find the global maximum.

\section{B. Review of Pillai's Method [1]}

Now we briefly review the method proposed in [1] for solving the optimization problem in (6). Note that the original method proposed in [1] uses a continuous-time SISO model. We review a slightly modified version of this method which works in our discrete-time MIMO model as shown in Fig. 1(b).

To solve (6), we can first solve $\mathbf{h}$ in terms of $\mathbf{f}$. In this case, the optimization problem becomes

$$
\max _{\mathbf{h}} \frac{\left|\mathbf{h}^{\dagger} \mathbf{T f}\right|^{2}}{\mathbf{h}^{\dagger} E\left[\mathbf{C f f} \mathbf{C}^{\dagger}\right] \mathbf{h}+\mathbf{h}^{\dagger} E\left[\mathbf{v v}{ }^{\dagger}\right] \mathbf{h}} .
$$

Define

$$
\mathbf{R}_{c, f} \triangleq E\left[\mathbf{C f f}^{\dagger} \mathbf{C}^{\dagger}\right]
$$

and $\mathbf{R}_{v}=E\left[\mathbf{v v}^{\dagger}\right]$. Note that $\mathbf{R}_{c, f}$ can be obtained by using the clutter covariance $\mathbf{R}_{c}(m)$ in (1) and $\mathbf{R}_{v}$ can be obtained by using the noise covariance $\mathbf{R}_{v}(m)$ in (2). The above problem can be recast as

$\min _{\mathbf{h}} \mathbf{h}^{\dagger}\left(\mathbf{R}_{c, f}+\mathbf{R}_{v}\right) \mathbf{h}$

subject to $\mathbf{h}^{\dagger} \mathbf{T f}=1$.

This is the well-known minimum variance distortionless response (MVDR) problem [37]. The solution to this problem is

$$
\mathbf{h}=\alpha\left(\mathbf{R}_{c, f}+\mathbf{R}_{v}\right)^{-1} \mathbf{T f} .
$$

where $\alpha$ is a scalar which satisfies the equality constraint. Note that the scalar can be ignored because it has no effect on the original objective function in (5).

Substituting the above $\mathbf{h}$ back into the objective function in (5). The new objective function becomes $\mathbf{f}^{\dagger} \mathbf{T}^{\dagger}\left(\mathbf{R}_{c, f}+\right.$ $\left.\mathbf{R}_{v}\right)^{-1} \mathbf{T} \mathbf{f}$ which is a function of $\mathbf{f}$ only. Therefore the optimization problem becomes

$$
\max _{\mathbf{f}} \mathbf{f}^{\dagger} \mathbf{T}^{\dagger}\left(\mathbf{R}_{c, f}+\mathbf{R}_{v}\right)^{-1} \mathbf{T f}
$$

$$
\text { subject to }\|\mathbf{f}\|^{2} \leq 1 .
$$

Now this problem has only one parameter $\mathbf{f}$. If $\mathbf{R}_{c, f}$ is a constant, the above problem is the well-known Rayleigh quotient [43] and the solution to $\mathbf{f}$ will be the principal component of the matrix $\mathbf{T}^{\dagger}\left(\mathbf{R}_{c, f}+\mathbf{R}_{v}\right)^{-1} \mathbf{T}$. However, note that from (7), $\mathbf{R}_{c, f}$ is a function of $\mathbf{f}$ as well. To solve this problem, Pillai et al. proposed a method which starts with an initial $\mathbf{f}$ and then uses this $\mathbf{f}$ to compute the matrix $\mathbf{T}^{\dagger}\left(\mathbf{R}_{c, f}+\mathbf{R}_{v}\right)^{-1} \mathbf{T}$. Then the principal component of this matrix is computed to update $\mathbf{f}$. This process is repeated until the SINR is large enough. As we have shown here, this method can be used in the MIMO case. Nevertheless, this method does not guarantee nondecreasing SINR in each iteration step. Consequently, the convergence cannot be guaranteed.

\section{PRoposed Iterative Method}

In this section, a new iterative algorithm is introduced for solving the SINR maximization problem in (6). Different from the approach in [1], this proposed method guarantees nondecreasing SINR in each iteration step. The technique applied here is that we first optimize the receiving filter $\mathbf{h}$ for fixed transmitted waveforms $\mathbf{f}$ and then optimize $\mathbf{f}$ for fixed receiving filter h. This kind of optimization technique has been applied in different fields such as multiuser transceiver design [32], multicarrier transceiver design [33], and adaptive paraunitary filterbank design [34]. It can be shown that the algorithm gives a solution which is not only a local optimum, but also the global optimum separately along the $\mathbf{f}$ dimension and the $\mathbf{h}$ dimension.

We have already solved $\mathbf{h}$ in terms of $\mathbf{f}$ in (8). Now we explain how to solve $\mathbf{f}$ in terms of $\mathbf{h}$ and then we will explain the iterative process. For fixed $\mathbf{h}$, the transmitted waveforms $\mathbf{f}$ can be obtained by solving the following optimization problem:

$$
\max _{\mathbf{f}} \frac{\left|\mathbf{h}^{\dagger} \mathbf{T f}\right|^{2}}{\mathbf{f}^{\dagger} \mathbf{R}_{c, h} \mathbf{f}+\mathbf{h}^{\dagger} \mathbf{R}_{v} \mathbf{h}} \text { subject to }\|\mathbf{f}\|^{2} \leq 1
$$

where $\mathbf{R}_{c, h} \triangleq E\left[\mathbf{C}^{\dagger} \mathbf{h} \mathbf{h}^{\dagger} \mathbf{C}\right]$ and $\mathbf{R}_{v} \triangleq E\left[\mathbf{v v} \mathbf{v}^{\dagger}\right]$. Note that both $\mathbf{R}_{c, h}$ and $\mathbf{R}_{v}$ can be obtained by using the prior second order information defined in (1) and (2). We first look at the Lagrange multiplier method to solve this problem. The Lagrangian can be defined as

$$
L(\mathbf{f}, \lambda) \triangleq \frac{\left|\mathbf{h}^{\dagger} \mathbf{T f}\right|^{2}}{\mathbf{f}^{\dagger} \mathbf{R}_{c, h} \mathbf{f}+\mathbf{h}^{\dagger} \mathbf{R}_{v} \mathbf{h}}+\lambda\left(\mathbf{f}^{\dagger} \mathbf{f}-1\right), \lambda \geq 0
$$

where $\lambda$ is the Lagrange multiplier. Differentiating the above function with respect to $f$ and setting it to zero, we obtain

$$
\frac{\mathbf{T}^{\dagger} \mathbf{h} \mathbf{h}^{\dagger} \mathbf{T f} \mathbf{f}\left(\mathbf{f}^{\dagger} \mathbf{R}_{c, h} \mathbf{f}+\mathbf{h}^{\dagger} \mathbf{R}_{v} \mathbf{h}\right)-\left|\mathbf{h}^{\dagger} \mathbf{T f}\right|^{2} \mathbf{R}_{c, h} \mathbf{f}}{\left(\mathbf{f}^{\dagger} \mathbf{R}_{c, h} \mathbf{f}+\mathbf{h}^{\dagger} \mathbf{R}_{v} \mathbf{h}\right)^{2}}+\lambda \mathbf{f}=0 .
$$

One can see that the above equation has a high order polynomial of $\mathbf{f}$ in the numerator. This makes it hard to solve in general.

We have already seen that directly solving the problem using the method of Lagrange multiplier is not easy. To overcome this difficulty, we recast the problem by using the following proposition.

Proposition 1: If $\mathbf{f}_{\star}$ solves the optimization problem

$$
\max _{f} \frac{\left|\mathbf{h}^{\dagger} \mathbf{T f}\right|^{2}}{\mathbf{f}^{\dagger} \mathbf{R}_{c, h} \mathbf{f}+\mathbf{h}^{\dagger} \mathbf{R}_{v} \mathbf{h} \cdot \mathbf{f}^{\dagger} \mathbf{f}}
$$

then $\mathbf{f}_{\star \star} \triangleq \mathbf{f}_{\star} /\left\|\mathbf{f}_{\star}\right\|$ solves $(10)$.

Proof: For any $\mathbf{f} \in \mathbb{C}^{N_{T}\left(L_{T}+1\right) \times 1}$ satisfying $\|\mathbf{f}\|^{2} \leq 1$

$$
\begin{aligned}
\frac{\left|\mathbf{h}^{\dagger} \mathbf{T} \mathbf{f}_{\star \star}\right|^{2}}{\mathbf{f}_{\star \star}^{\dagger} \mathbf{R}_{c, h} \mathbf{f}_{\star \star}+\mathbf{h}^{\dagger} \mathbf{R}_{v} \mathbf{h}} & =\frac{\left|\mathbf{h}^{\dagger} \mathbf{T} \mathbf{f}_{\star}\right|^{2}}{\mathbf{f}_{\star}^{\dagger} \mathbf{R}_{c, h} \mathbf{f}_{\star}+\mathbf{h}^{\dagger} \mathbf{R}_{v} \mathbf{h} \cdot \mathbf{f}_{\star}^{\dagger} \mathbf{f}_{\star}} \\
& \geq \frac{\left|\mathbf{h}^{\dagger} \mathbf{T f}\right|^{2}}{\mathbf{f}^{\dagger} \mathbf{R}_{c, h} \mathbf{f}+\mathbf{h}^{\dagger} \mathbf{R}_{v} \mathbf{h} \cdot \mathbf{f}^{\dagger} \mathbf{f}} \\
& \geq \frac{\left|\mathbf{h}^{\dagger} \mathbf{T f}\right|^{2}}{\mathbf{f}^{\dagger} \mathbf{R}_{c, h} \mathbf{f}+\mathbf{h}^{\dagger} \mathbf{R}_{v} \mathbf{h}} .
\end{aligned}
$$

The first inequality is because of the definition of $\mathbf{f}_{\star}$. The second inequality is from the fact that $\|\mathbf{f}\|^{2} \leq 1$. We also have $\left\|\mathbf{f}_{\star \star}\right\|^{2} \leq$ 1 . Therefore $f_{\star \star \star}$ is a solution to (10). 
Similar technique has been used in [32] to solve an MSE minimization problem in multiuser transceivers. This proposition allows us to get rid of the power constraint in (10) and solve the unconstrained problem in (11) instead. Equation (11) can be further recast as the MVDR problem

$\min _{\mathbf{f}} \mathbf{f}^{\dagger}\left(\mathbf{R}_{c, h}+\mathbf{h}^{\dagger} \mathbf{R}_{v} \mathbf{h} \cdot \mathbf{I}\right) \mathbf{f}$

subject to $\mathbf{h}^{\dagger} \mathbf{T f}=1$.

The solution to the above problem is [37]

$$
\mathbf{f}=\alpha\left(\mathbf{R}_{c, h}+\mathbf{h}^{\dagger} \mathbf{R}_{v} \mathbf{h} \cdot \mathbf{I}\right)^{-1} \mathbf{T}^{\dagger} \mathbf{h}
$$

where $\alpha$ is a scalar which satisfies the power constraint. Note that this scalar can be ignored because $f$ needs to be normalized to unit norm according to Proposition 1.

Now we know how to solve $\mathbf{h}$ in terms of $\mathbf{f}$ and $\mathbf{f}$ in terms of $\mathbf{h}$. We can iteratively solve for the transmitted waveforms $\mathbf{f}$ and the receiving filter $\mathbf{h}$. Thus the objective function, namely SINR, will be nondecreasing in every iteration step. The algorithm is summarized as follows.

Algorithm 1: Given the target impulse response $\mathbf{T}(z)$, noise covariance $\mathbf{R}_{v}(m)$, the clutter covariance $\mathbf{R}_{c}(m)$, and an initial value of the transmitted waveforms $\mathbf{f}$, the transceiver pair $(\mathbf{f}, \mathbf{h})$ can be optimized by repeating the following steps:

1. Compute $\mathbf{R}_{c, f}=E\left[\mathbf{C f f}^{\dagger} \mathbf{C}^{\dagger}\right]$

2. $\mathbf{h} \leftarrow\left(\mathbf{R}_{c, f}+\mathbf{R}_{v}\right)^{-1} \mathbf{T f}$

3. Compute $\mathbf{R}_{c, h}=E\left[\mathbf{C}^{\dagger} \mathbf{h h}{ }^{\dagger} \mathbf{C}\right]$

4. $\mathbf{f} \leftarrow\left(\mathbf{R}_{c, h}+\mathbf{h}^{\dagger} \mathbf{R}_{v} \mathbf{h} \cdot \mathbf{I}\right)^{-1} \mathbf{T}^{\dagger} \mathbf{h}$

5. $\mathbf{f} \leftarrow \mathbf{f} /\|\mathbf{f}\|$.

We stop when the SINR improvement becomes insignificant. Because the objective function is bounded and is nondecreasing in each step, according to monotone convergence theorem the objective function will converge to some value $\Phi_{\star}$ [46]. Even though the point $(\mathbf{f}, \mathbf{h})$ corresponding to this result $\Phi_{\star}$ is not unique, the algorithm stops in one particular $\left(\mathbf{f}_{\star}, \mathbf{h}_{\star}\right)$ yielding $\Phi_{\star}$. Since $\mathbf{f}_{\star}$ and $\mathbf{h}_{\star}$ are local optimum along $\mathbf{f}$ and $\mathbf{h}$ dimension separately, the solution $\left(\mathbf{f}_{\star}, \mathbf{h}_{\star}\right)$ is also a local optimum, that is

$$
\begin{aligned}
\exists \epsilon & >0 \text { such that } \\
\rho\left(\mathbf{f}_{\star}, \mathbf{h}_{\star}\right) & \geq \rho(\mathbf{f}, \mathbf{h}), \forall\left\|\mathbf{f}-\mathbf{f}_{\star}\right\|^{2}+\left\|\mathbf{h}-\mathbf{h}_{\star}\right\|^{2} \leq \epsilon .
\end{aligned}
$$

Moreover, the algorithm finds the global maximum along $\mathbf{f}$ dimension or $\mathbf{h}$ dimension in each step. Therefore, when the algorithm converges, the solution $\left(\mathbf{f}_{\star}, \mathbf{h}_{\star}\right)$ will be the global optimum along the $\mathbf{f}$ dimension and $\mathbf{h}$ dimension separately, that is

$$
\begin{aligned}
& \rho\left(\mathbf{f}_{\star}, \mathbf{h}_{\star}\right) \geq \rho\left(\mathbf{f}_{\star}, \mathbf{h}\right), \forall \mathbf{h} \\
& \rho\left(\mathbf{f}_{\star}, \mathbf{h}_{\star}\right) \geq \rho\left(\mathbf{f}, \mathbf{h}_{\star}\right), \forall\|\mathbf{f}\|^{2} \leq 1 .
\end{aligned}
$$

So, the solution obtained by this iterative algorithm is actually stronger than a local maximum.

1) Matched Filter Bound: To evaluate the performance of the suboptimal iterative algorithm, we are interested in how close its SINR performance is to the global optimal solution of the problem in (6). However, it does not appear to be a simple matter to obtain the global optimal solution. To avoid this difficulty, we compare the performance of the proposed method to a computable upper bound of the global maximum. One way to obtain such an upper bound is to drop the clutter term in the SINR expression in (5). This bound is often called the matched filter bound. It can be expressed as

$$
\max _{\mathbf{f}, \mathbf{h}} \frac{\left|\mathbf{h}^{\dagger} \mathbf{T f}\right|^{2}}{\mathbf{h}^{\dagger} \mathbf{R}_{v} \mathbf{h}} \text { subject to }\|\mathbf{f}\|^{2} \leq 1 \text {. }
$$

One can first solve $\mathbf{h}$ in terms of $\mathbf{f}$ as in (8) and obtain

$$
\mathbf{h}=\alpha \mathbf{R}_{v}^{-1} \mathbf{T} \mathbf{f}
$$

where $\alpha$ is a scalar which will be determined by the power constraint. Substituting the above solution into the objective function, the optimization problem becomes

$$
\max _{\mathbf{f}} \mathbf{f}^{\dagger} \mathbf{T}^{\dagger} \mathbf{R}_{v}^{-1} \mathbf{T f} \text { subject to }\|\mathbf{f}\|^{2} \leq 1 .
$$

This is the well-known Rayleigh quotient [43]. The solution of $\mathbf{f}$ is the principal component of the matrix $\mathbf{T}^{\dagger} \mathbf{R}_{v}^{-1} \mathbf{T}$ and the maximum of the objective function is the largest eigenvalue of $\mathbf{T}^{\dagger} \mathbf{R}_{v}^{-1} \mathbf{T}$ which is denoted as

$$
\lambda_{1}\left(\mathbf{T}^{\dagger} \mathbf{R}_{v}^{-1} \mathbf{T}\right) .
$$

Therefore, this matched filter bound can be easily obtained. The numerical results for the proposed iterative method and the matched filter bound will be presented in Section V.

\section{ITERATIVE METHOD With RANDOM AND UNCERTAIN TARGET IMPULSE RESPONSE}

The iterative method introduced in Section III requires the information of the target impulse response $\mathbf{T}(z)$. In this section, we focus on the case where only a partial information of the target impulse response is available. We consider two different cases. In the first, the target impulse response is modelled as a WSS random process. We assume only the covariance of the process is known. An iterative algorithm for maximizing the SINR in this case will be derived in this section. In the second case, the target impulse response is deterministic but unknown. We assume the target impulse response lies in a convex uncertainty set. An iterative algorithm will be proposed in this section to maximize the worst SINR among all the possible target impulse responses in the given uncertainty set.

\section{A. Random Target Impulse Response}

We first consider the random target impulse response case. We assume the coefficients of the target impulse response $\operatorname{vec}(\mathbf{t}(n))$ is a WSS random process. We assume the covariance matrix, which is defined as

$$
\mathbf{R}_{t}(m) \triangleq E\left[\operatorname{vec}(\mathbf{t}(n)) \operatorname{vec}(\mathbf{t}(n-m))^{\dagger}\right]
$$

is known. In this case, the SINR at the receiving filter output is defined as

$$
\rho(\mathbf{f}, \mathbf{h}) \triangleq \frac{E\left[\left|\mathbf{h}^{\dagger} \mathbf{T f}\right|^{2}\right]}{E\left[\left|\mathbf{h}^{\dagger} \mathbf{C f}\right|^{2}\right]+E\left[|\mathbf{h} \mathbf{v}|^{2}\right]} .
$$


The goal is to solve the following optimization problem:

$$
\max _{\mathbf{f}, \mathbf{h}} \rho(\mathbf{f}, \mathbf{h}) \text { subject to }\|\mathbf{f}\|^{2} \leq 1 \text {. }
$$

The same technique used in Section III can be used to iteratively optimize the parameters $\mathbf{h}$ and $\mathbf{f}$ in each step. To solve $\mathbf{h}$ in terms of $\mathbf{f}$, the optimization problem can be written as

$$
\max _{\mathbf{h}} \frac{\mathbf{h}^{\dagger} \mathbf{R}_{t, f} \mathbf{h}}{\mathbf{h}^{\dagger} \mathbf{R}_{c, f} \mathbf{h}+\mathbf{h}^{\dagger} \mathbf{R}_{v} \mathbf{h}}
$$

where $\mathbf{R}_{t, f} \triangleq E\left[\mathbf{T f f}^{\dagger} \mathbf{T}^{\dagger}\right], \mathbf{R}_{c, f} \triangleq E\left[\mathbf{C f f}^{\dagger} \mathbf{C}^{\dagger}\right]$ and $\mathbf{R}_{v} \triangleq$ $E\left[\mathbf{v v}{ }^{\dagger}\right]$. Define $\mathbf{L}_{c, f}$ as the lower triangular Cholesky factor of $\mathbf{R}_{c, f}+\mathbf{R}_{v}$. In other words, the lower triangular matrix $\mathbf{L}_{c, f}$ satisfies $\mathbf{L}_{c, f} \mathbf{L}_{c, f}^{\dagger}=\mathbf{R}_{c, f}+\mathbf{R}_{v}$. Define $\mathbf{x} \triangleq \mathbf{L}_{c, f}^{\dagger} \mathbf{h}$. By changing variables, the optimization problem can be rewritten as

$$
\max _{\mathbf{x}} \frac{\mathbf{x}^{\dagger} \mathbf{L}_{c, f}^{-1} \mathbf{R}_{t, f} \mathbf{L}_{c, f}^{-\dagger} \mathbf{x}}{\mathbf{x}^{\dagger} \mathbf{x}}
$$

This is the well-known Rayleigh quotient [43] and the solution to the problem is the principal component of the matrix $\mathbf{L}_{c, f}^{-1} \mathbf{R}_{t, f} \mathbf{L}_{c, f}$. Thus, the solution $\mathbf{h}$ can be expressed as

$$
\mathbf{h}=\mathbf{L}_{c, f}^{-\dagger} \cdot \mathbf{p}\left(\mathbf{L}_{c, f}^{-1} \mathbf{R}_{t, f} \mathbf{L}_{c, f}^{-\dagger}\right)
$$

where $\mathbf{p}(\mathbf{A})$ denotes the principal component of matrix $\mathbf{A}$.

To solve $\mathbf{f}$ in terms of $\mathbf{h}$, the optimization problem becomes the following:

$\max _{\mathbf{f}} \frac{\mathbf{f}^{\dagger} \mathbf{R}_{t, h} \mathbf{f}}{\mathbf{f}^{\dagger} \mathbf{R}_{c, h} \mathbf{f}+\mathbf{h}^{\dagger} \mathbf{R}_{v} \mathbf{h}}$

subject to $\|\mathbf{f}\|^{2} \leq 1$

where $\mathbf{R}_{t, h} \triangleq E\left[\mathbf{T}^{\dagger} \mathbf{h} \mathbf{h}^{\dagger} \mathbf{T}\right]$ and $\mathbf{R}_{c, h} \triangleq E\left[\mathbf{C}^{\dagger} \mathbf{h} \mathbf{h}^{\dagger} \mathbf{C}\right]$. It can be easily verified that a similar result as in Proposition 1 still holds in this case. We can obtain the solution by solving the following problem instead:

$$
\max _{\mathbf{f}} \frac{\mathbf{f}^{\dagger} \mathbf{R}_{t, h} \mathbf{f}}{\mathbf{f}^{\dagger} \mathbf{R}_{c, h} \mathbf{f}+\mathbf{h}^{\dagger} \mathbf{R}_{v} \mathbf{h} \cdot \mathbf{f}^{\dagger} \mathbf{f}} .
$$

Using the same technique for solving $\mathbf{h}$, we can obtain

$$
\mathbf{f}=\mathbf{L}_{c, f}^{-\dagger} \cdot \mathbf{p}\left(\mathbf{L}_{c, f}^{-1} \mathbf{R}_{t, h} \mathbf{L}_{c, f}^{-\dagger}\right)
$$

where $\mathbf{L}_{c, f}$ is the lower triangular matrix such that $\mathbf{L}_{c, f} \mathbf{L}_{c, f}^{\dagger}=$ $\mathbf{R}_{c, h}+\mathbf{h}^{\dagger} \mathbf{R}_{v} \mathbf{h} \cdot \mathbf{I}$.

We summarize the iterative algorithm for optimizing the transceiver pair in the case of random target impulse response as the following.

Algorithm 2: Given the target impulse response covariance $\mathbf{R}_{t}(m)$, the clutter covariance $\mathbf{R}_{c}(m)$, the noise covariance $\mathbf{R}_{v}(m)$, and an initial value of the transmitted waveforms $\mathbf{f}$, the transceiver pair $(\mathbf{f}, \mathbf{h})$ can be optimized by repeating the following steps:

1. Compute $\mathbf{R}_{c, f}=E\left[\mathrm{Cff}^{\dagger} \mathbf{C}^{\dagger}\right], \mathbf{R}_{t, f}=E\left[\mathbf{T f f}^{\dagger} \mathbf{T}^{\dagger}\right]$
2. Compute the Cholesky decomposition $\mathbf{R}_{c, f}+\mathbf{R}_{v}=$ $\mathbf{L}_{c, f} \mathbf{L}_{c, f}^{\dagger}$

3. $\mathbf{h} \leftarrow \mathbf{L}_{c, f}^{-\dagger} \mathbf{p}\left(\mathbf{L}_{c, f}^{-1} \mathbf{R}_{t, f} \mathbf{L}_{c, f}^{-\dagger}\right)$

4. Compute $\mathbf{R}_{c, h}=E\left[\mathbf{C}^{\dagger} \mathbf{h h}{ }^{\dagger} \mathbf{C}\right], \mathbf{R}_{t, h}=E\left[\mathbf{T}^{\dagger} \mathbf{h h}{ }^{\dagger} \mathbf{T}\right]$

5. Compute the Cholesky decomposition $\mathbf{R}_{c, h}+\mathbf{h}^{\dagger} \mathbf{R}_{v} \mathbf{h} \cdot \mathbf{I}=$ $\mathbf{L}_{c, f} \mathbf{L}_{c, f}^{\dagger}$

6. $\mathbf{f} \leftarrow \mathbf{L}_{c, f}^{-\dagger} \mathbf{p}\left(\mathbf{L}_{c, f}^{-1} \mathbf{R}_{t, h} \mathbf{L}_{c, f}^{-\dagger}\right)$

7. $\mathbf{f}=\mathbf{f} /\|\mathbf{f}\|$

We stop when the SINR improvement becomes insignificant.

We have extended the proposed iterative method to the random target impulse response case. Note that it is not clear how to extend the method proposed in [1] to this case. The method proposed in [1] requires substituting the solution of $\mathbf{h}$ in (8) back into the objective function and obtain the optimization problem with only $\mathbf{f}$ parameter as in (9). In the random target impulse response case, substituting the solution $\mathbf{h}$ in (14) back, the objective function becomes

$$
\lambda_{1}\left(\mathbf{L}_{c, f}^{-1} \mathbf{R}_{t, f} \mathbf{L}_{c, f}^{-\dagger}\right)
$$

where $\lambda_{1}(\mathbf{A})$ denotes the maximum eigenvalue of matrix $\mathbf{A}$. The iterative method proposed in [1] treats the matrix $\mathbf{R}_{c, f}$ in (9) as a constant with respect to $\mathbf{f}$ in every iteration and obtains the transmitted waveforms $\mathbf{f}$ by Rayleigh principle [43]. However, in the case of random target impulse response, even if we treat the matrix $\mathbf{L}_{c, f}^{-1}$ as a constant with respect to $\mathbf{f}$, it does not appear to be a simple matter to choose $\mathbf{f}$ to maximize the objective function in (15). For similar reason, it does not appear to be a simple matter to compute the matched filter bound which we have obtained for the deterministic target case.

\section{B. Uncertain Target Impulse Response}

We now consider the second case where the target impulse response is deterministic but unknown. We assume the target matrix $\mathbf{T}$ lies in a known convex set $\mathcal{S}$. Our goal is to maximize the worst SINR in this set. The worst SINR can be expressed as

$$
\rho(\mathbf{f}, \mathbf{h})=\min _{\mathbf{T} \in \mathcal{S}} \frac{\left|\mathbf{h}^{\dagger} \mathbf{T f}\right|^{2}}{E\left[\left|\mathbf{h}^{\dagger} \mathbf{C f}\right|^{2}\right]+E\left[|\mathbf{h} \dagger \mathbf{v}|^{2}\right]} .
$$

So the goal is to solve the following optimization problem:

$$
\max _{\mathbf{f}, \mathbf{h}} \rho(\mathbf{f}, \mathbf{h}) \text { subject to }\|\mathbf{f}\|^{2} \leq 1 \text {. }
$$

To solve this problem, we first recast the problem using the following proposition.

Proposition 2: Define

$$
\eta(\mathbf{f}, \mathbf{h}) \triangleq \min _{\mathbf{T} \in \mathcal{S}} \frac{\left|\mathbf{h}^{\dagger} \mathbf{T f}\right|^{2}}{E\left[\left|\mathbf{h}^{\dagger} \mathbf{C f}\right|^{2}\right]+E\left[\left|\mathbf{h}^{\dagger} \mathbf{v}\right|^{2}\right] \cdot \mathbf{f}^{\dagger} \mathbf{f}}
$$

If $\left(\mathbf{f}_{\star}, \mathbf{h}_{\star}\right)$ solves the problem

$$
\max _{\mathbf{f}, \mathbf{h}} \eta(\mathbf{f}, \mathbf{h})
$$

then $\left(\mathbf{f}_{\star \star}, \mathbf{h}_{\star}\right)$ solves $(17)$, where $\mathbf{f}_{\star \star} \triangleq \mathbf{f}_{\star} /\left\|\mathbf{f}_{\star}\right\|$. 
Proof: For any $(\mathbf{f}, \mathbf{h}) \in \mathbb{C}^{N_{T}\left(L_{T}+1\right)} \times \mathbb{C}^{N_{R}\left(L_{R}+1\right)}$ satisfying $\|\mathbf{f}\|^{2} \leq 1$, we have $\rho\left(\mathbf{f}_{\star \star}, \mathbf{h}\right)=\eta\left(\mathbf{f}_{\star}, \mathbf{h}\right) \geq \boldsymbol{\eta}(\mathbf{f}, \mathbf{h}) \geq$ $\rho(\mathbf{f}, \mathbf{h})$. Also, $\left\|\mathbf{f}_{\star \star}\right\|^{2} \leq 1$. Therefore, $\left(\mathbf{f}_{\star \star}, \mathbf{h}_{\star}\right)$ solves (17).

One can see that the logical flow of this proof is identical to Proposition 1. This proposition allows us to get rid of the power constraint in (17) and solve the unconstrained problem in (19) instead. To solve the max-min problem, one can first solve for the worst target matrix $\mathbf{T}$ in the minimization problem in (18). Since the feasible set $\mathcal{S}$ is convex and the objective function is quadratic with respect to $\mathbf{T}$, the optimization problem in (18) is a convex problem. It can be solved numerically if the values of $\mathbf{f}$ and $\mathbf{h}$ are given. However, the values of $\mathbf{f}$ and $\mathbf{h}$ have not yet been given at this moment. They are parameters which will be maximized in (19). So, in order to solve the problem in this manner, we need to solve $\mathbf{T}$ in terms of $\mathbf{f}$ and $\mathbf{h}$ analytically. Then the objective function $\eta(\mathbf{f}, \mathbf{h})$ in (18) can be expressed analytically in terms of $\mathbf{f}$ and $\mathbf{h}$. However, in general, the analytic solution may not be available. Even if we can obtain the analytic form of $\mathbf{T}$, the resulting function $\eta(\mathbf{f}, \mathbf{h})$ might not be concave in terms of parameter $\mathbf{f}$ or $\mathbf{h}$. If $\eta(\mathbf{f}, \mathbf{h})$ is not concave in terms of $\mathbf{f}$ or $\mathbf{h}$, the problem in (19) is in general not easy to solve.

To overcome this difficulty, we apply the following proposition.

Proposition 3: If $\left(\mathbf{x}_{\star}, \mathbf{T}_{\star}\right)$ solves

$$
\min _{\mathbf{T} \in \mathcal{S}} \max _{\mathbf{x}} \frac{\left|\mathbf{y}^{\dagger} \mathbf{T} \mathbf{x}\right|^{2}}{\mathbf{x}^{\dagger} \mathbf{R x}}
$$

for some $\mathbf{y}$ and $\mathbf{R}$, then it also solves

$$
\max _{\mathbf{x}} \min _{\mathbf{T} \in \mathcal{S}} \frac{\left|\mathbf{y}^{\dagger} \mathbf{T} \mathbf{x}\right|^{2}}{\mathbf{x}^{\dagger} \mathbf{R x}}
$$

Proof: Applying Proposition 1 in [35], one can verify that $\left(\mathbf{x}_{\star}, \mathbf{T}_{\star}\right)$ is a saddle point, that is

$$
\begin{aligned}
& \frac{\left|\mathbf{y}^{\dagger} \mathbf{T}_{\star} \mathbf{x}\right|^{2}}{\mathbf{x}^{\dagger} \mathbf{R x}} \leq \frac{\left|\mathbf{y}^{\dagger} \mathbf{T}_{\star} \mathbf{x}_{\star}\right|^{2}}{\mathbf{x}_{\star}^{\dagger} \mathbf{R} \mathbf{x}_{\star}} \\
& \leq \frac{\left|\mathbf{y}^{\dagger} \mathbf{T} \mathbf{x}_{\star}\right|^{2}}{\mathbf{x}_{\star}^{\dagger} \mathbf{R} \mathbf{x}_{\star}}, \forall \mathbf{x} \neq 0, \mathbf{T} \in \mathcal{S} .
\end{aligned}
$$

By using the minimax theorem [44], the saddle point also solves the second optimization problem.

This proposition allows us to change the order of the maximization with respect $\mathbf{f}$ and $\mathbf{h}$ and the minimization with respect to $\mathbf{T}$ in (18) and (19).

To solve the optimization problem in (19), we use the iterative approach as before. In each step, we optimize $\mathbf{f}$ with fixed $\mathbf{h}$ or optimize $\mathbf{h}$ with fixed $\mathbf{f}$. We first demonstrate how to solve $\mathbf{f}$ with fixed $\mathbf{h}$, that is, to solve

$$
\max _{\mathbf{f}} \min _{\mathbf{T} \in \mathcal{S}} \frac{\left|\mathbf{h}^{\dagger} \mathbf{T f}\right|^{\dagger}}{\mathbf{f}^{\dagger} \mathbf{R}_{c, h} \mathbf{f}+\mathbf{h}^{\dagger} \mathbf{R}_{v} \mathbf{h} \cdot \mathbf{f}^{\dagger} \mathbf{f}}, \text { for fixed } \mathbf{h} .
$$

Applying Proposition 3, the above problem can be recast as

$$
\min _{\mathbf{T} \in S} \max _{\mathbf{f}} \frac{\left|\mathbf{h}^{\dagger} \mathbf{T f}\right|^{2}}{\mathbf{f}^{\dagger}\left(\mathbf{R}_{c, h}+\mathbf{h}^{\dagger} \mathbf{R}_{v} \mathbf{h} \cdot \mathbf{I}\right) \mathbf{f}} .
$$

Using the same MVDR approach for obtaining (12), one can obtain

$$
\mathbf{f}=\alpha\left(\mathbf{R}_{c, h}+\mathbf{h}^{\dagger} \mathbf{R}_{v} \mathbf{h} \cdot \mathbf{I}\right)^{-1} \mathbf{T}^{\dagger} \mathbf{h}
$$

where $\alpha$ is a scalar which will be determined by the power constraint. Substituting $\mathbf{f}$ into the objective function, the optimization problem becomes

$$
\min _{\mathbf{T} \in \mathcal{S}} \mathbf{h}^{\dagger} \mathbf{T}\left(\mathbf{R}_{c, h}+\mathbf{h}^{\dagger} \mathbf{R}_{v} \mathbf{h} \cdot \mathbf{I}\right)^{-1} \mathbf{T}^{\dagger} \mathbf{h} .
$$

Observing the above problem, one can see that the cost function is a convex function and the feasible set $\mathcal{S}$ is a convex set. Therefore it is a convex optimization problem. Note that since $\mathbf{h}$ is fixed now, the solution $\mathbf{T}$ can be solved numerically. This $\mathbf{T}$ yields the worst case target in the uncertainty set.

With similar technique, one can also solve $\mathbf{h}$ with fixed $\mathbf{f}$, and obtain the following solution:

$$
\mathbf{h}=\alpha\left(\mathbf{R}_{c, f}+\mathbf{R}_{v}\right)^{-1} \mathbf{T f}
$$

where $\alpha$ is a scalar which will be determined by the power constraint, and $\mathbf{T}$ is the solution to the following convex optimization problem

$$
\min _{\mathbf{T} \in \mathcal{S}} \mathbf{f}^{\dagger} \mathbf{T}^{\dagger}\left(\mathbf{R}_{c, f}+\mathbf{R}_{v}\right)^{-1} \mathbf{T f}
$$

which can be solved numerically.

With these methods, we can increase the worst SINR defined in (16) in each step by optimizing $\mathbf{f}$ or $\mathbf{h}$ one at a time. We summarize the algorithm as the following.

Algorithm 3: Given the target matrix uncertainty set $\mathcal{S}$, the clutter covariance $\mathbf{R}_{c}(m)$, noise covariance $\mathbf{R}_{v}(m)$, and an initial value of the transmitted waveforms $\mathbf{f}$, the transceiver pair $(\mathbf{f}, \mathbf{h})$ can be optimized by the following steps.

$$
\begin{aligned}
& \text { 1. Compute } \mathbf{R}_{c, f}=E\left[\mathbf{C f f}^{\dagger} \mathbf{C}^{\dagger}\right] \\
& \text { 2. } \mathbf{T}_{\star} \leftarrow \arg \min _{\mathbf{T} \in \mathcal{S}} \mathbf{f}^{\dagger} \mathbf{T}^{\dagger}\left(\mathbf{R}_{c, f}+\mathbf{R}_{v}\right)^{-1} \mathbf{T f} \\
& \text { 3. } \mathbf{h} \leftarrow\left(\mathbf{R}_{c, f}+\mathbf{R}_{v}\right)^{-1} \mathbf{T}_{\star} \mathbf{f} \\
& \text { 4. Compute } \mathbf{R}_{c, h}=E\left[\mathbf{C}^{\dagger} \mathbf{h h} \mathbf{h}^{\dagger} \mathbf{C}\right] \\
& \text { 5. } \mathbf{T}_{\star} \leftarrow \arg \min _{\mathbf{T} \in \mathcal{S}} \mathbf{h}^{\dagger} \mathbf{T}\left(\mathbf{R}_{c, h}+\mathbf{h}^{\dagger} \mathbf{R}_{v} \mathbf{h} \cdot \mathbf{I}\right)^{-1} \mathbf{T}^{\dagger} \mathbf{h} \\
& \text { 6. } \mathbf{f} \leftarrow\left(\mathbf{R}_{c, h}+\mathbf{h}^{\dagger} \mathbf{R}_{v} \mathbf{h} \cdot \mathbf{I}\right)^{-1} \mathbf{T}_{\star}^{\dagger} \mathbf{h} \\
& \text { 7. } \mathbf{f} \leftarrow \mathbf{f} /\|\mathbf{f}\|
\end{aligned}
$$

We stop when the SINR improvement becomes insignificant.

1) Special Case: Sphere Uncertainty Set: Now we consider a sphere uncertainty set, that is

$$
\mathcal{S}=\left\{\mathbf{T} \mid\left\|\mathbf{T}-\mathbf{T}_{0}\right\|_{F}^{2} \leq r^{2}\right\}
$$

where $\mathbf{T}_{0}$ is the origin and $r$ is the radius of the sphere. Note that this is a special case of the convex uncertainty set. We assume $r$ is small enough so that $\mathbf{0} \notin \mathcal{S}$. This is a reasonable assumption because if $\mathbf{0} \in \mathcal{S}$, no matter how we choose $\mathbf{f}$ and $\mathbf{h}$, the worst SINR in (16) will always be zero. In this case, one can use the Lagrange multiplier method to solve for the worst SINR in (16) and obtain

$$
\eta(\mathbf{f}, \mathbf{h})=\frac{\left|\mathbf{h}^{\dagger} \mathbf{T}_{0} \mathbf{f}-r\|\mathbf{h}\|\|\mathbf{f}\| e^{j \measuredangle\left(\mathbf{h}^{\dagger} \mathbf{T}_{0} \mathbf{f}\right)}\right|^{2}}{E\left[\left|\mathbf{h}^{\dagger} \mathbf{C f}\right|^{2}\right]+\mathbf{h}^{\dagger} \mathbf{R}_{v} \mathbf{h}} .
$$


The derivation of the above worst SINR expression is shown in the Appendix . Although an analytic solution for the worst SINR can be obtained, one can see that $\eta(\mathbf{f}, \mathbf{h})$ is not convex in either $\mathbf{f}$ or $\mathbf{h}$. So it is difficult to maximize the worst SINR from the above expression.

We can apply Algorithm 3 to solve this problem. In this case, the convex optimization in Step 2 of Algorithm 3 becomes

$$
\min _{\left\|\mathbf{T}-\mathbf{T}_{0}\right\|_{F}^{2} \leq r^{2}} \mathbf{f}^{\dagger} \mathbf{T}^{\dagger}\left(\mathbf{R}_{c, f}+\mathbf{R}_{v}\right)^{-1} \mathbf{T f} .
$$

As we have mentioned earlier, this is a convex optimization problem and therefore can be solved numerically. However, we will show that in this special case of the sphere uncertainty set, a simple line search algorithm can be used to solve this problem. Define the Lagrangian as

$$
\begin{aligned}
L(\mathbf{T}, \lambda) \triangleq \mathbf{f}^{\dagger} \mathbf{T}^{\dagger}\left(\mathbf{R}_{c, f}+\right. & \left.\mathbf{R}_{v}\right)^{-1} \mathbf{T f} \\
& +\lambda\left(\operatorname{tr}\left(\left(\mathbf{T}-\mathbf{T}_{0}\right)\left(\mathbf{T}-\mathbf{T}_{0}\right)^{\dagger}\right)-r^{2}\right)
\end{aligned}
$$

where $\lambda \geq 0$ is the Lagrange multiplier. Differentiating the above function with respect to $\mathbf{T}$ and setting it to zero, we obtain

$$
\left(\mathbf{R}_{c, f}+\mathbf{R}_{v}\right)^{-1} \mathbf{T f f}{ }^{\dagger}+\lambda\left(\mathbf{T}-\mathbf{T}_{0}\right)=\mathbf{0} .
$$

From the above equation, one can observe that $\mathbf{T}-\mathbf{T}_{0}$ has rank one. Without loss of generality, there exists some $\mathbf{u} \in$ $\mathbb{C}^{N_{R}\left(L_{R}+1\right) \times 1}$ such that

$$
\mathbf{T}-\mathbf{T}_{0}=\mathbf{u f}^{\dagger}
$$

Substituting $\mathbf{T}=\mathbf{T}_{0}+\mathbf{u} \mathbf{f}^{\dagger}$ into (23) and solving for $\mathbf{u}$, one can obtain

$$
\mathbf{T}=\mathbf{T}_{0}-\left(\lambda\left(\mathbf{R}_{c, f}+\mathbf{R}_{v}\right)+\|f\|^{2} \mathbf{I}\right)^{-1} \mathbf{T}_{0} \mathrm{ff}^{\dagger} .
$$

We have almost finished solving for $\mathbf{T}$ except that there is still an unknown Lagrange multiplier $\lambda$ in the above equation. Note that usually the constraint in (22) can be either an active constraint

$$
\left\|\mathbf{T}-\mathbf{T}_{0}\right\|_{F}^{2}=r^{2}
$$

or an inactive constraint $\left\|\mathbf{T}-\mathbf{T}_{0}\right\|_{F}^{2}<r^{2}$. The inactive constraint only happens when the cost function reaches global minimum, that is, $\mathbf{T}=\mathbf{0}$. But $\mathbf{T}=\mathbf{0}$ cannot happen because we have assumed $\mathbf{0} \notin \mathcal{S}$. Consequently, the constraint will always be active as in (24). Thus the Lagrange multiplier $\lambda$ can be obtained by solving (24). Define the eigenvalue decomposition

$$
\mathbf{R}_{c, f}+\mathbf{R}_{v}=\mathrm{QDQ}^{\dagger}
$$

where $\mathbf{Q}$ is unitary and $\mathbf{D}$ is diagonal. Equation (24) can thus be expressed as

$$
\begin{aligned}
\| \mathbf{T} & -\mathbf{T}_{0} \|_{F}^{2} \\
& =\left\|\left(\lambda\left(\mathbf{R}_{c, f}+\mathbf{R}_{v}\right)+\|\mathbf{f}\|^{2} \mathbf{I}\right)^{-1} \mathbf{T}_{0} \mathrm{ff}^{\dagger}\right\|_{F}^{2} \\
& =\|\mathbf{f}\|^{2} \cdot\left\|\left(\lambda \mathbf{D}+\|\mathbf{f}\|^{2} \mathbf{I}\right)^{-1} \mathbf{Q}^{\dagger} \mathbf{T}_{0} \mathbf{f}\right\|^{2} \\
& =\|\mathbf{f}\|^{2} \cdot \sum_{i=0}^{N_{R}\left(L_{R}+1\right)-1} \frac{\left|\left(\mathbf{Q}^{\dagger} \mathbf{T}_{0} \mathbf{f}\right)_{i}\right|^{2}}{\left(\lambda \cdot(\mathbf{D})_{i, i}+\|\mathbf{f}\|^{2}\right)^{2}}=r^{2} .
\end{aligned}
$$

Note that $(\mathbf{D})_{i} \geq 0$ because $\mathbf{R}_{c, f}+\mathbf{R}_{v}$ is positive semidefinite. Also, we have the Lagrange multiplier $\lambda \geq 0$. Therefore, the left side of the last equality is a decreasing function of $\lambda$. This implies the solution for $\lambda$ is unique. In this case, the solution for $\lambda$ can be easily found by some simple line search algorithm such as Newton's method [45].

Using the same argument, one can solve the convex optimization problem in Step 5 of Algorithm 3 and obtain the following solution:

$$
\mathbf{T}=\mathbf{T}_{0}-\mathbf{h h}^{\dagger} \mathbf{T}_{0}\left(\lambda\left(\mathbf{R}_{c, h}+\mathbf{h}^{\dagger} \mathbf{R}_{v} \mathbf{h} \cdot \mathbf{I}\right)+\|\mathbf{h}\|^{2} \mathbf{I}\right)^{-1}
$$

where $\lambda$ is the Lagrange multiplier. It can be solved by a line search algorithm using the following relation

$$
\|\mathbf{h}\|^{2} \cdot \sum_{i=0}^{N_{T}\left(L_{T}+1\right)-1} \frac{\left|\left(\mathbf{Q} \mathbf{T}_{0}^{\dagger} \mathbf{h}\right)_{i}\right|^{2}}{\left(\lambda \cdot(\mathbf{D})_{i, i}+\|\mathbf{h}\|^{2}\right)^{2}}=r^{2}
$$

where $\mathrm{QDQ}^{\dagger}$ is the eigenvalue decomposition of the matrix $\left(\mathbf{R}_{c, h}+\mathbf{h}^{\dagger} \mathbf{R}_{v} \mathbf{h} \cdot \mathbf{I}\right)$.

Therefore, in the sphere uncertainty set case, the numerical convex optimization in Step 2 and Step 5 of Algorithm 3 can be replaced by the aforementioned method which can be much more efficient. In [36], similar result has been obtained for solving beamformer robust against steering vector mismatch. The second order cone programming has been replaced by the line search algorithm in [36], by using the Lagrange multiplier method.

\section{NuMERICAL RESULTS}

In this section, the SINR performances of the proposed method are compared to the method in [1] which has been extended to the MIMO case in [14], and to the orthogonal LFM (linear frequency modulation) waveforms. The orthogonal LFM waveforms is defined as

$$
(\mathbf{f})_{l}=\frac{e^{j \pi 1 / 2\left(L_{T}+1\right)\left(l \bmod N_{T}\right)^{2}} \cdot e^{j 2 \pi\left\lfloor l / N_{T}\right\rfloor\left(\operatorname{lmod} N_{T}\right)}}{\sqrt{N_{T}\left(L_{T}+1\right)}} .
$$

Note that LFM waveforms are designed for a different purpose, namely, obtaining a sharp ambiguity function. They are good candidates for distinguish point targets, and in imaging. However, the LFM waveforms may not have good SINR performances in the extended target case.

1) Example 1: SINR Versus Number of Iterations: Consider a MIMO radar system with number of transmitting antennas $N_{T}=4$ and number of receiving antennas $N_{R}=4$. The target impulse response is given by

$$
(\mathbf{t}(n))_{k, l}= \begin{cases}1, & n=0,1, \ldots, 20 \\ 0, & \text { otherwise }\end{cases}
$$

The clutter impulse response is modelled as an AR (auto-regressive) process with covariance

$$
\mathbf{R}_{c}(n)=\mathbf{B A}^{|n|} \mathbf{B}^{\dagger}
$$

where the parameters $\mathbf{A}$ and $\mathbf{B}$ are $16 \times 16$ real matrices shown in Fig. 3. Here $\mathbf{A}$ is a positive semidefinite matrix with spectral radius less than unity. The noise $\mathbf{v}(n)$ is modelled as white noise with unity variance. Fig. 4 shows the SINR performances 


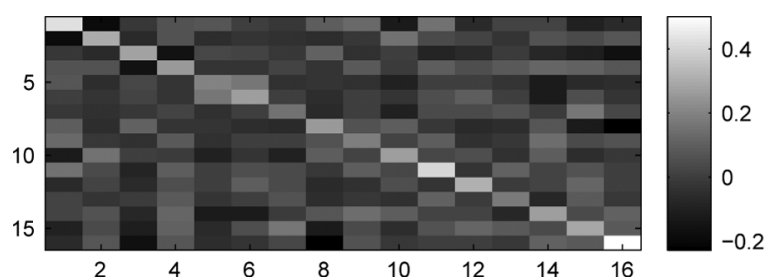

(a)

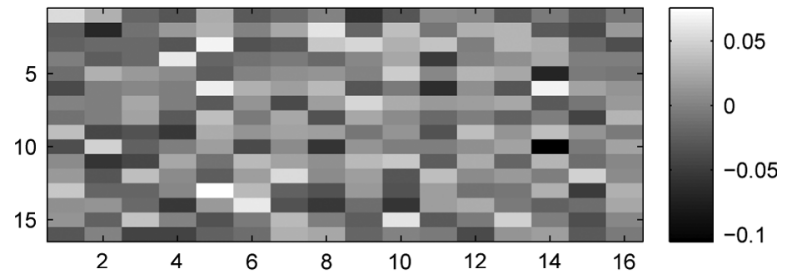

(b)

Fig. 3. Example 1: The parameters used in the matrix AR model (a) Matrix A. (b) Matrix $\mathbf{B}$

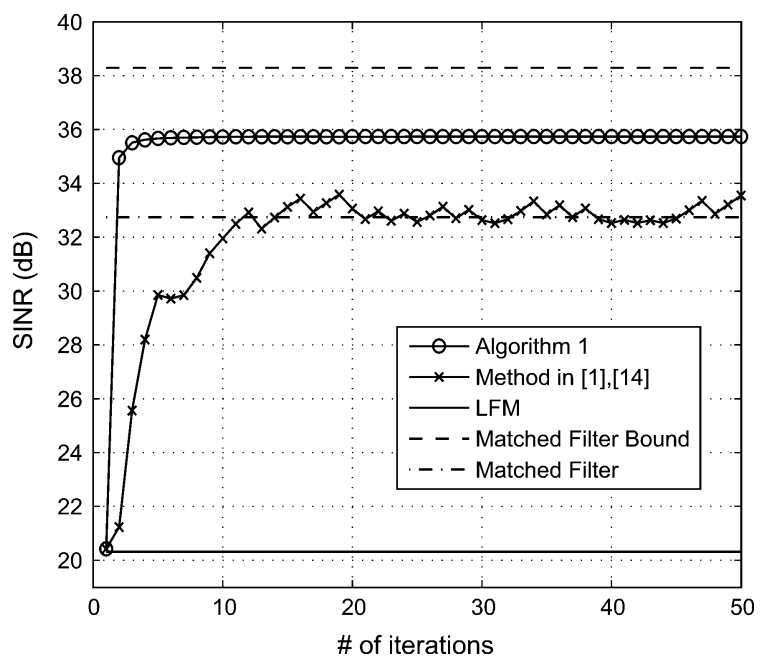

Fig. 4. Example 1: Comparison of the SINR versus number of iterations.

defined in (5) as a function of the number of iterations. The matched filter bound which has been derived in the end of Section III is also shown in the figure. The matched filter SINR, which takes the clutter signal into account is also shown in the figure. The Note that LFM waveform is fixed, so its SINR is not a function of the number of iterations. The initial waveforms used in Algorithm 1 (the new algorithm proposed in Section III) and in the method in [1] and [14] are identical. One can observe that Algorithm 1 has a better performance than other methods. Algorithm 1 also converges very fast. It converges in about six iterations in this example. Moreover, in Algorithm 1, the SINR is a nondecreasing function of the number of iterations. The initial transmitted waveforms are shown in Fig. 5(a)-(d). Note that the vector $\mathbf{f}$ contains four waveforms as defined in (3). The optimized transmitted waveforms and receiving filters are shown in Fig. 5(e)-(1).

2) Example 2: SINR Versus Clutter-to-Noise Ratio (CNR): In this example, the SINR performances are compared for different values of CNR. Consider a MIMO radar with number of transmitting antennas $N_{T}=2$ and number of receiving antennas $N_{R}=2$. The order of the target impulse response $\mathbf{T}(z)$ is 20 .

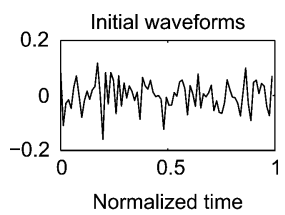

(a)

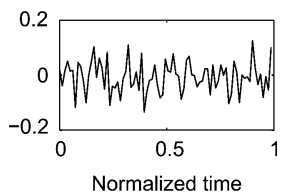

(b)

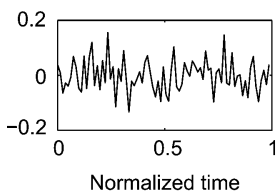

(c)

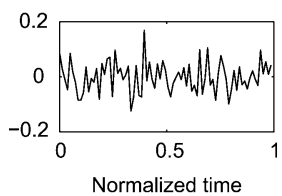

(d)

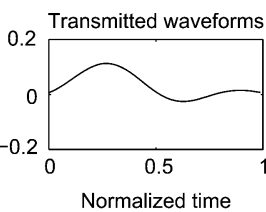

(e)

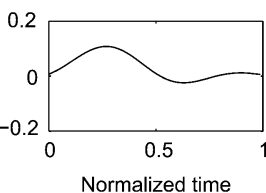

(f)

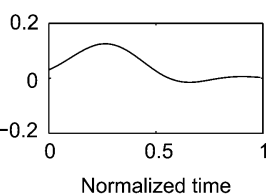

(g)

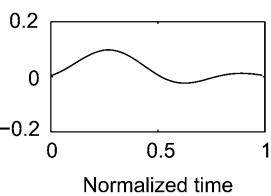

(h)

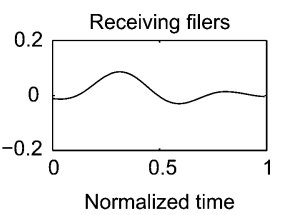

(i)

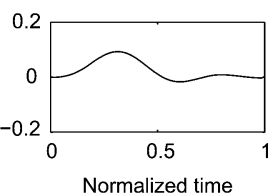

(j)

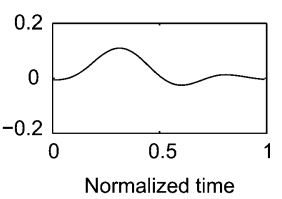

(k)

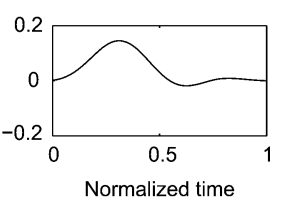

(I)
Fig. 5. Example 1: (a)-(d) Real part of the initial transmitted waveforms. (e)-(h) Real part of the transmitted four waveforms $\mathbf{f}$ obtained by Algorithm 1. (i)-(l) Real part of the four receiving filters $\mathbf{h}$ obtained by Algorithm 1.

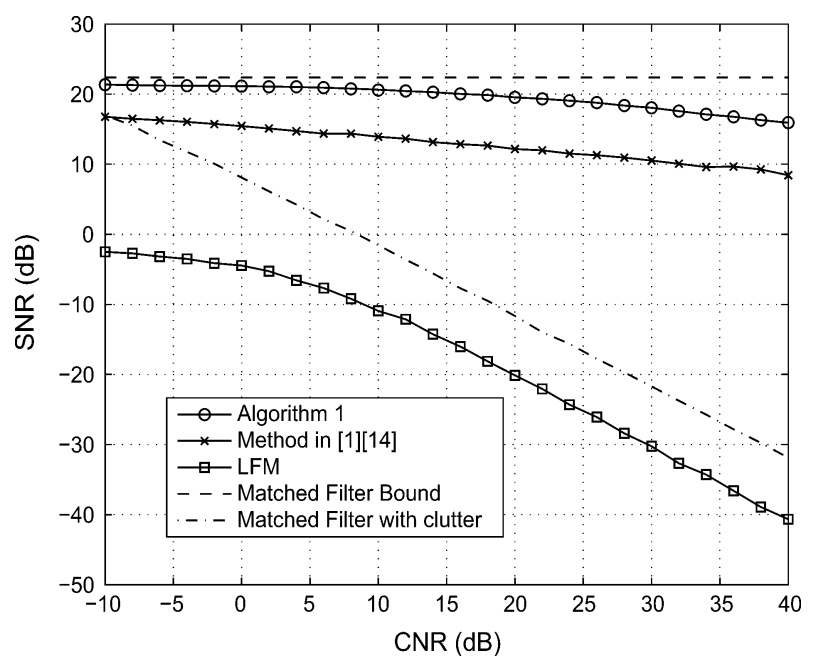

Fig. 6. Example 2: Comparison of the SINR versus CNR.

The coefficients $\left\{(\mathbf{t}(n))_{k, l}\right\}$ are generated as i.i.d. (independent and identically distributed) circular complex Gaussian random variables with unity variance. The covariance of the clutter impulse response $\mathbf{R}_{c}(n)$ is generated by using

$$
\mathbf{R}_{c}(n)=\mathbf{U}_{c}(n) * \mathbf{U}_{c}(-n)^{\dagger}
$$

where the notation $*$ denotes convolution, $\mathbf{U}_{c}(n)$ is a $4 \times 4$ matrix sequence with length 31 and the coefficients $\left\{(\mathbf{U}(n))_{k, l}\right\}$ are i.i.d. circular complex Gaussian random variables. The noise $\mathbf{v}(n)$ is a white process with unity variance. The initial waveforms used in the algorithms are randomly chosen. The simulation is performed by averaging among 1000 different target, clutter and noise realizations. Fig. 6 shows the comparison of 


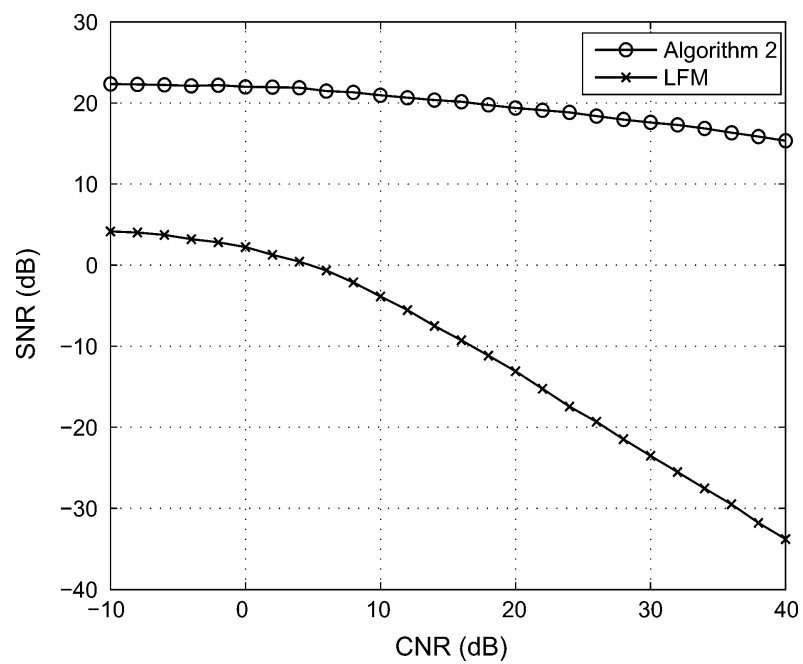

Fig. 7. Example 3: Comparison of the SINR versus CNR with random target impulse response.

the SINR defined in (5) under different CNR. The matched filter bound which has been derived in the end of Section III is also shown on the figure. The matched filter SINR, which takes the clutter signal into account is also shown in the figure. One can see that Algorithm 1 has the best SINR performances among all the methods under all CNR. Both Algorithm 1 and method in [1] and [14] have much better performances than the LFM waveforms. This shows that utilizing the prior information in the transmitter is very crucial for the SINR performance.

3) Example 3: SINR Versus CNR With Random Target Impulse Response: In this example, the SINR performances are compared under different CNR as in the last example. However, the coefficients of the target impulse response $\operatorname{vec}(\mathbf{t}(n))$ are modelled as a WSS random process with covariance $\mathbf{R}_{t}(n)$. The covariance $\mathbf{R}_{t}(n)$ is generated by using

$$
\mathbf{R}_{t}(n)=\mathbf{U}_{t}(n) * \mathbf{U}_{t}(-n)^{\dagger}
$$

where $\mathbf{U}_{t}(n)$ is a $4 \times 4$ matrix sequence with length 21 and the coefficients $\left\{\left(\mathbf{U}_{t}(n)\right)_{k, l}\right\}$ are i.i.d. circular complex Gaussian random variables. Except the target impulse response, all the parameters used in this example are identical to Example 2. The simulation is performed by averaging among 1000 different target, clutter, and noise realizations. Fig. 7 shows the comparison of the SINR defined in (5) under various CNR. We have explained in Section IV-A that it is not clear how to generalize the method in [1] and [14] and the matched filter bound to this case. Thus we only compare Algorithm 2 and LFM waveforms in this case. One can see that Algorithm 2 has a significantly better SINR performances than the LFM waveforms.

4) Example 4: Worst SINR Versus CNR With Uncertain Target Impulse Response: In this example, we consider that the target matrix $\mathbf{T}$ is in a sphere uncertainty set as shown in (20). The worst SINR in (21) are compared under various CNR. All the parameters are identical to the last example, except the target impulse response. The center $\mathbf{T}_{0}$ of the sphere is a block Toeplitz matrix generated by the matrix sequence $\mathbf{t}_{0}(n)$ as in (4). The order of $\mathbf{t}_{0}(n)$ is 20 . The elements of $\mathbf{t}_{0}(n)$,

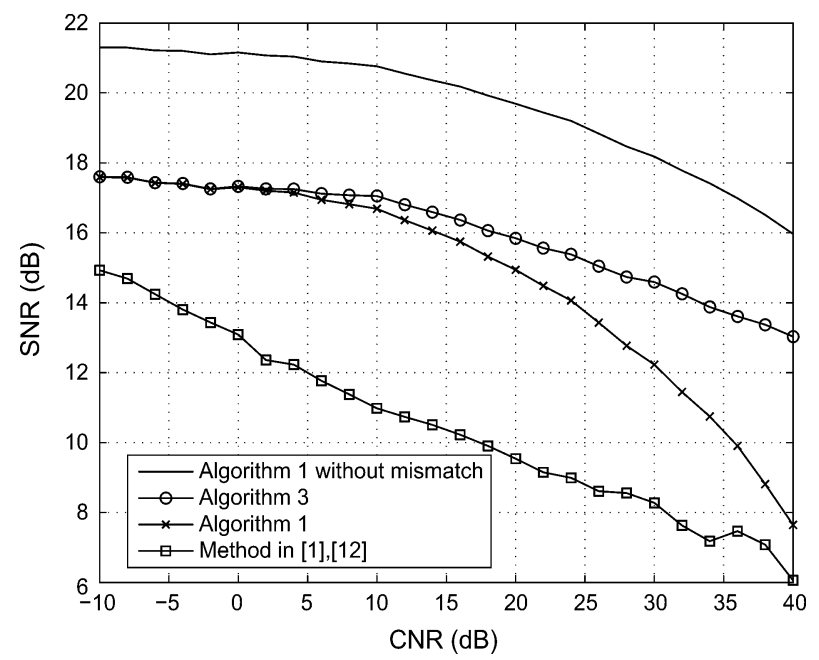

Fig. 8. Example 4: Comparison of the worst SINR versus CNR with uncertain target impulse response.

namely $\left\{\left(\mathbf{t}_{0}(n)\right)_{k, l}\right\}$, are generated as i.i.d. circular complex Gaussian random variables with unity variance. The radius $r$ is chosen to be $5 \%$ of $\|\mathbf{T}\|_{F}$. The simulation is performed by averaging among 1000 different target center $\mathbf{T}_{0}$, clutter, and noise realizations. In this example, the following four different SINR results are compared:

1) Algorithm 1 without mismatch: The transceiver pair $(\mathbf{f}, \mathbf{h})$ is obtained by using Algorithm 1 with the target matrix $\mathbf{T}=\mathbf{T}_{0}$. The SINR is obtained by using (5) with $\mathbf{T}=\mathbf{T}_{0}$ as well.

2) Algorithm 3: The transceiver is obtained by using Algorithm 3 with the origin $\mathbf{T}_{0}$ and radius $r$. The SINR is the worst SINR obtained by using (16).

3) Algorithm 1: The transceiver is obtained by using Algorithm 1 with the target matrix $\mathbf{T}_{0}$. The SINR is the worst SINR obtained by using (16).

4) Method in [1], [14]: The transceiver is obtained by using the method in [1] and [14] with the target matrix $\mathbf{T}_{0}$. The SINR is the worst SINR obtained by using (16).

Fig. 8 shows the SINR performances under different CNR. As expected, Algorithm 1 without the target mismatch has the best SINR performance. Algorithm 3 which is designed for robustness against target mismatch has a significantly better worst SINR performance compared to Algorithm 1 in the high CNR region.

\section{CONCLUSION}

In this paper, we have proposed an iterative algorithm for jointly designing the transmitted waveforms and the receiving filters to maximize the SINR in MIMO radar with the prior information of the extended target and clutter. This iterative algorithm alternatively solves the optimal transmitted waveforms and the receiving filters by fixing the other parameters. This algorithm finds a local maximum which is also a global maximum along the dimension of the transmitted waveforms and the dimension of the receiving filter separately. The proposed iterative algorithm has also been extended to the case of random target impulse response and the case of uncertain target impulse 
response. The numerical results show that the proposed iterative algorithm converges faster and also has better SINR performances than previously reported algorithms. Some of the important design issues such as constant modulus and range resolution are not considered in this paper. From a practical stand point, it is important that the radar transmitter has a low PAR. Also, the optimal waveforms obtained by the proposed method may not have good performance for range estimation. The waveform design problem which takes into account these important issues will be explored in the future.

\section{APPENDIX}

Here, we derive the worst SINR in the case of sphere uncertainty target impulse response as shown in (21). For simplicity, we ignore the irrelevant denominator in (16). The following optimization problem is considered

$$
\min _{\mathbf{T}}\left|\mathbf{h}^{\dagger} \mathbf{T f}\right|^{2} \quad \text { subject to }\left\|\mathbf{T}-\mathbf{T}_{0}\right\|_{F}^{2} \leq r^{2} .
$$

The Lagrangian of the above problem can be defined as

$$
L(\mathbf{T}, \lambda) \triangleq\left|\mathbf{h}^{\dagger} \mathbf{T} \mathbf{f}\right|^{2}+\lambda\left(\operatorname{tr}\left(\left(\mathbf{T}-\mathbf{T}_{0}\right)\left(\mathbf{T}-\mathbf{T}_{0}\right)^{\dagger}\right)-r^{2}\right)
$$

where $\lambda \geq 0$ is the Lagrange multiplier. Differentiating the Lagrangian with respect to $\mathbf{T}$ and setting it to zero, we obtain

$$
\left(\mathbf{h}^{\dagger} \mathbf{T f}\right) \cdot \mathbf{h f}^{\dagger}+\lambda\left(\mathbf{T}-\mathbf{T}_{0}\right)=\mathbf{0} .
$$

From the above equality, without loss of generality, there exists a scalar $\alpha$ such that

$$
\mathbf{T}-\mathbf{T}_{0}=\alpha \mathbf{h f}^{\dagger} .
$$

Note that usually the constraint in (25) can be either an active constraint

$$
\left\|\mathbf{T}-\mathbf{T}_{0}\right\|_{F}^{2}=r^{2}
$$

or an inactive constraint $\left\|\mathbf{T}-\mathbf{T}_{0}\right\|_{F}^{2}<r^{2}$. The inactive constraint only happens when the cost function reaches global minimum, that is, $\mathbf{T}=\mathbf{0}$. But $\mathbf{T}=\mathbf{0}$ cannot happen because we have assumed $\mathbf{0} \notin \mathcal{S}$. Consequently, the constraint will always be active as in (27). By substituting (26) into (27), one can obtain the magnitude of $\alpha$ as

$$
|\alpha|=\frac{r}{\|\mathbf{h}\|\|\mathbf{f}\|} .
$$

Substituting this result back into (26), we obtain

$$
\mathbf{T}=\mathbf{T}_{0}-\frac{r \mathbf{h f} \dagger^{\dagger}}{\|\mathbf{f}\|\|\mathbf{h}\|} e^{j \measuredangle \alpha} .
$$

Now the only unknown in the above equation is the phase $\measuredangle \alpha$. To solve for the unknown phase, substituting the above expression into the cost function, one can obtain

$$
\left|\mathbf{h}^{\dagger} \mathbf{T f}\right|^{2}=\left|\mathbf{h}^{\dagger} \mathbf{T}_{0} \mathbf{f}-r\left\|\mathbf{h}||\left|\mathbf{f} \| e^{j \measuredangle \alpha}\right|^{2} .\right.\right.
$$

One can easily verify that the phase which minimizes the above cost function is $\measuredangle \alpha=\measuredangle \mathbf{h}^{\dagger} \mathbf{T}_{0}$ f. Therefore the solution to the problem in (27) is

$$
\mathbf{T}=\mathbf{T}_{0}-\frac{r \mathbf{h} \mathbf{f}^{\dagger}}{\|\mathbf{f}\|\|\mathbf{h}\|} e^{j \measuredangle\left(\mathbf{h}^{\dagger} \mathbf{T}_{0} \mathbf{f}\right)} .
$$

Substituting the above solution back into the cost function, one can obtain the minimum as

$$
\left|\mathbf{h}^{\dagger} \mathbf{T} \mathbf{f}\right|^{2}=\left|\mathbf{h}^{\dagger} \mathbf{T}_{0} \mathbf{f}-r\|\mathbf{h}\|\|\mathbf{f}\| e^{j \measuredangle\left(\mathbf{h}^{\dagger} \mathbf{T}_{0} \mathbf{f}\right)}\right|^{2} .
$$

\section{REFERENCES}

[1] S. U. Pillai, H. S. Oh, D. C. Youla, and J. R. Guerci, "Optimal transmitreceiver design in the presence of signal-dependent interference and channel noise," IEEE Trans. Inf. Theory, vol. 46, no. 2, pp. 577-584, Mar. 2003.

[2] D. DeLong and E. Hofstetter, "On the design of optimum radar waveforms for clutter rejection," IEEE Trans. Inf. Theory, vol. 13, no. 3, pp. 454-463, Jul. 1967.

[3] D. Delong and E. Hofstertter, "The design of clutter-resistant radar waveforms with limited dynamic range," IEEE Trans. Inf. Theory, vol. 15, no. 3, pp. 376-385, May 1969.

[4] D. Delong and E. Hofstertter, "Design of radar signals and receivers subject to implementation errors," IEEE Trans. Inf. Theory, vol. 16, no. 6, pp. 707-711, Nov. 1970.

[5] D. R. Fuhrmann and G. S. Antonio, "Transmit beamforming for MIMO radar systems using partial signal correlation," in Proc. 38th IEEE Asilomar Conf. Signals, Syst., Comput., Nov. 2004, pp. 295-299.

[6] D. R. Fuhrmann and G. S. Antonio, "Transmit beamforming for MIMO radar systems using signal cross-correlation," IEEE Trans. Aerosp. Electron. Syst., vol. 44, pp. 171-186, Jan. 2008.

[7] P. Stoica, J. Li, and Y. Xie, "On probing signal design for MIMO radar," IEEE Trans. Signal Process., vol. 55, no. 8, pp. 4151-4161, Aug. 2007.

[8] J. Li, L. Xu, P. Stoica, K. W. Forsythe, and D. Bliss, "Range compression and waveform optimization for MIMO radar: A Cramér-Rao bound based study," IEEE Trans. Signal Process., vol. 56, no. 1, pp. 218-232, Jan. 2008.

[9] P. Stoica, J. Li, and X. Zhu, "Waveform synthesis for diversity-based transmit beampattern design," IEEE Trans. Signal Process., Jun. 2008.

[10] C. Y. Chen and P. P. Vaidyanathan, "MIMO radar ambiguity properties and optimization using frequency-hopping waveforms," IEEE Trans. Signal Process., Dec. 2008.

[11] J. Li, P. Stoica, and X. Zheng, "Signal synthesis and receiver design for MIMO radar imaging," IEEE Trans. Signal Process., Aug. 2008.

[12] B. Liu, Z. He, J. Zeng, and B. Y. Liu, "Polyphase orthogonal code design for MIMO radar systems," in Proc. Int. Conf. Radar, Oct. 2006, pp. $1-4$.

[13] B. Liu, Z. He, and Q. He, "Optimization of orthogonal discrete frequency-coding waveform based on modified genetic algorithm for MIMO radar," in Proc. Int. Conf. Commun., Circuits, Syst., Jul. 2007, pp. 966-970.

[14] B. Friedlander, "Waveform design for MIMO radars," IEEE Trans. Aerosp. Electron. Syst., vol. 43, pp. 1227-1238, Jul. 2007.

[15] Y. Yang and R. S. Blum, "MIMO radar waveform design based on mutual information and minimum mean-square error estimation," IEEE Trans. Aerosp. Electron. Syst., vol. 43, no. 1, pp. 330-343, Jan. 2007.

[16] Y. Yang and R. S. Blum, "Minimax robust MIMO radar waveform design," IEEE J. Sel. Topics Signal Process., vol. 1, no. 1, pp. 147-155, Jun. 2007.

[17] J. Li, J. R. Guerci, and L. Xu, "Signal waveform's optimal under restriction design for active sensing," IEEE Signal Process. Lett., Sep. 2006.

[18] A. De Maio, S. D. Nicola, Y. Huang, S. Zhang, and A. Farina, "Code design to optimize radar detection performance under accuracy and similarity constraints," IEEE Trans. Signal Process., Nov. 2008.

[19] M. R. Bell, "Information theory and radar waveform design," IEEE Trans. Inf. Theory, vol. 39, no. 5, pp. 1578-1597, Sep. 1993. 
[20] A. Leshem, O. Naparstek, and A. Nehorai, "Information theoretic adaptive radar waveform design for multiple extended targets," IEEE J. Sel. Topics Signal Process., vol. 1, no. 1, pp. 42-55, Jun. 2007.

[21] D. J. Rabideau and P. Parker, "Ubiquitous MIMO multifunction digital array radar," in Proc. 37th IEEE Asilomar Conf. Signals, Syst., Comput., Nov. 2003, vol. 1, pp. 1057-1064.

[22] D. W. Bliss and K. W. Forsythe, "Multiple-input multiple-output (MIMO) radar and imaging: Degrees of freedom and resolution," in Proc. 37th IEEE Asilomar Conf. Signals, Syst., Comput., Nov. 2003, vol. 1, pp. 54-59.

[23] E. Fishler, A. M. Haimovich, R. S. Blum, D. Chizhik, L. J. Cimini, and R. A. Valenzuela, "MIMO radar: An idea whose time has come," in Proc. IEEE Radar Conf., Apr. 2004, pp. 71-78.

[24] E. Fishler, A. M. Haimovich, R. S. Blum, L. J. Cimini, D. Chizhik, and R. A. Valenzuela, "Performance of MIMO radar systems: Advantages of angular diversity," in Proc. 38th IEEE Asilomar Conf. Signals, Syst., Comput., Nov. 2004, vol. 1, pp. 305-309.

[25] C. Duofang, C. Baixiao, and Q. Guodong, "Angle estimation using esprit in MIMO radar," Electron. Lett., vol. 44, no. 12, pp. 770-771, Jun. 2008.

[26] E. Fishler, A. M. Haimovich, R. S. Blum, L. J. Cimini, D. Chizhik, and R. A. Valenzuela, "Spactial diversity in radars-models and detection performance," IEEE Trans. Signal Process., vol. 54, no. 3, pp. 823-837, Mar. 2006.

[27] A. M. Haimovich, R. S. Blum, and L. J. Cimini, "MIMO radar with widely separated antennas," IEEE Signal Process. Mag., vol. 25, no. 1, pp. 116-129, Jan. 2008.

[28] J. Li and P. Stoica, "MIMO radar with colocated antennas," IEEE Signal Process. Mag., vol. 24, no. 5, Sep. 2007.

[29] J. Li, P. Stoica, L. Xu, and W. Roberts, "On parameter identifiability of MIMO radar," IEEE Signal Process. Lett., vol. 14, Dec. 2007.

[30] C. Y. Chen and P. P. Vaidyanathan, "MIMO radar space-time adaptive processing using prolate spheroidal wave functions," IEEE Trans. Signal Process., vol. 56, no. 2, Feb. 2008.

[31] V. F. Mecca, D. Ramakrishnan, and J. L. Krolik, "MIMO radar space-time adaptive processing for multipath clutter mitigation," in IEEE Workshop on Sens. Array and Multichannel Signal Process., Jul. 2006, pp. 249-253.

[32] S. Serbetli and A. Yener, "Transceiver optimization for multiuser MIMO systems," IEEE Trans. Signal Process., vol. 52, no. 1, pp. 214-226, Jan. 2004.

[33] S. M. Phoong, Y. B. Chang, and C. Y. Chen, "DFT-modulated filterbank transceivers for multipath fading channels," IEEE Trans. Signal Process., vol. 53, no. 1, pp. 182-192, Jan. 2005.

[34] A. Tkacenko and P. P. Vaidyanathan, "Iterative greedy algorithm for solving the FIR paraunitary approximation problem," IEEE Trans. Signal Process., vol. 54, no. 1, pp. 146-160, Jan. 2006.

[35] S. J. Kim, A. Magnani, A. Mutapcic, S. P. Boyd, and Z. Q. Luo, "Robust beamforming via worst-case SINR maximization," IEEE Trans. Signal Process., vol. 56, no. 4, pp. 1539-1547, Apr. 2008.

[36] R. G. Lorenz and S. P. Boyd, "Robust minimum variance beamforming," IEEE Trans. Signal Process., vol. 53, pp. 1684-1696, May 2005.

[37] J. Capon, "High-resolution frequency-wavenumber spectrum analysis," Proc. IEEE, vol. 57, pp. 1408-1418, Aug. 1969.

[38] G. San Antonio, D. R. Fuhrmann, and F. C. Robey, "MIMO radar ambiguity functions," IEEE J. Sel. Topics in Signal Process., vol. 1, pp. 167-177, Jun. 2007.

[39] Y. I. Abramovich and G. J. Frazer, "Bounds on the volume and height distributions for the MIMO radar ambiguity function," IEEE Signal Process. Lett., vol. 15, pp. 505-508.

[40] J. Y. Qu, J. Y. Zhang, and C. Q. Liu, "The ambiguity function of MIMO radar," in Proc. Int. Symp. Microw., Antenna, Propag. EMC Technol. Wireless Commun., Aug. 2007, pp. 265-268.

[41] N. Levanon and E. Mozeson, Radar Signals. New York: Wiley-IEEE, 2004.

[42] H. L. Van Tree, Detection, Estimation, and Modulation Theory, Part I. New York: Wiley-Interscience, 2001.

[43] R. A. Horn and C. R. Johnson, Matrix Analysis. Cambridge, U.K.: Cambridge Univ. Press, 1990.

[44] M. Willem, Minimax Theorems. Boston, MA: Birkhäuser, 1997.
[45] S. Boyd and L. Vandenberghe, Convex Optimization. Cambridge, U.K.: Cambridge Univ. Press, 2004.

[46] J. Yeh, "Real analysis," in Theory of measure and integration. Singapore: World Scientific, 2006.

[47] C. Y. Chen and P. P. Vaidyanathan, "Joint MIMO radar waveform and receiving filter optimization," in Proc. Int. Conf. Acoust., Speech Signal Process., Taipei, Taiwan R.O.C, Apr. 2009.

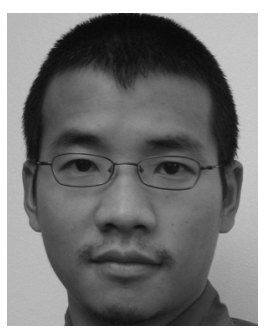

Chun-Yang Chen (S'05) was born in Taipei, Taiwan, on Nov. 22, 1977. He received the B.S. and M.S. degrees in electrical engineering and communication engineering, both from National Taiwan University (NTU), Taipei, Taiwan, in 2000 and 2002, respectively.

$\mathrm{He}$ is currently pursuing the Ph.D. degree in electrical engineering in the field of digital signal processing at California Institute of Technology (Caltech), Pasadena. His interests currently include signal processing in MIMO communications, ultrawideband communications, and radar applications.

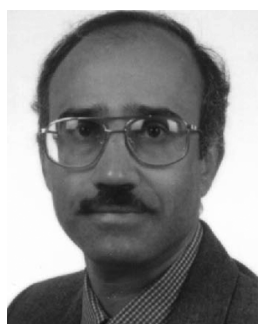

P. P. Vaidyanathan ( $\left.\mathrm{S}^{\prime} 80-\mathrm{M}^{\prime} 83-\mathrm{SM}^{\prime} 88-\mathrm{F}^{\prime} 91\right)$ was born in Calcutta, India, on October 16, 1954. He received the B.Sc. (Hons.) degree in physics and the B.Tech. and M.Tech. degrees in radiophysics and electronics, all from the University of Calcutta, in 1974, 1977, and 1979, respectively. He received the $\mathrm{Ph} . \mathrm{D}$ degree in electrical and computer engineering from the University of California, Santa Barbara, in 1982.

He was a Postdoctoral Fellow with the University of California, Santa Barbara, from September 1982 to March 1983. In March 1983, he joined the Electrical Engineering Department, California Institute of Technology (Caltech), Pasadena, as an Assistant Professor, and since 1993, has been Professor of electrical engineering. His main research interests are in digital signal processing, multirate systems, wavelet transforms, and signal processing for digital communications. He has authored a number of papers in IEEE journals, and is the author of the book Multirate Systems and Filter Banks (Englewood Cliffs, NJ: Prentice-Hall, 1993). He has written several chapters for various signal processing handbooks.

Dr. Vaidyanathan served as Vice Chairman of the Technical Program Committee for the 1983 IEEE International Symposium on Circuits and Systems, and as the Technical Program Chairman for the 1992 IEEE International Symposium on Circuits and Systems. He was an Associate Editor for the IEEE TRANSACTIONS ON CIRCUITS AND SYSTEMS during 1985-1987, and is currently an Associate Editor for the IEEE Signal PROCESSING LeTters, and a consulting editor for the Applied and Computational Harmonic Analysis. He was Guest Editor in 1998 for special issues of the IEEE TRANSACTIONS ON SIGNAL PROCESSING and the IEEE TRANSACTIONS ON CIRCUITS AND SYSTEMS II, on the topics of filter banks, wavelets, and subband coders. He was a recipient of the Award for excellence in teaching at the California Institute of Technology for the years 1983-1984, 1992-1993, and 1993-1994. He also received the NSF's Presidential Young Investigator award in 1986. In 1989, he received the IEEE ASSP Senior Award for his paper on multirate perfect-reconstruction filter banks. In 1990, he was the recipient of the S. K. Mitra Memorial Award from the Institute of Electronics and Telecommunications Engineers, India, for his joint paper in the IETE journal. He was also the coauthor of a paper on linear-phase perfect reconstruction filter banks in the IEEE TRANSACTIONS ON SIGNAL PROCESSING, for which the first author (T. Nguyen) received the Young Outstanding Author Award in 1993. He received the 1995 F. E. Terman Award of the American Society for Engineering Education, sponsored by Hewlett Packard Co., for his contributions to engineering education, especially Multirate Systems And Filter Banks. He has given several plenary talks including at the Sampta'01, Eusipco'98, SPCOM'95, and Asilomar'88 conferences on signal processing. He has been chosen a Distinguished Lecturer for the IEEE Signal Processing Society for 1996-1997. In 1999, he received the IEEE CAS Society's Golden Jubilee Medal. In 2002, he received the IEEE Signal Processing Society's Technical Achievement Award. 